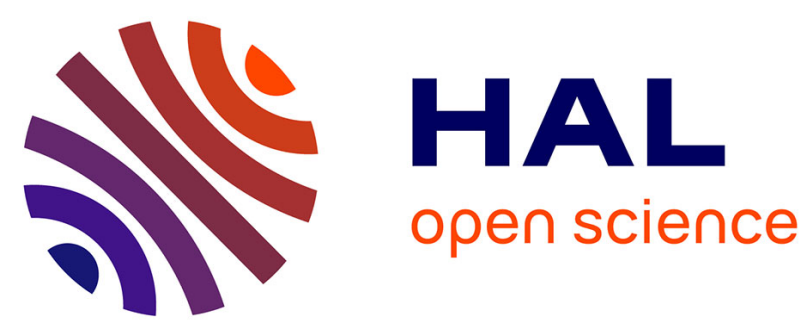

\title{
The dynamic modular fingerprints of the human brain at rest
}

\author{
Aya Kabbara, Veronique Paban, Mahmoud Hassan
}

\section{To cite this version:}

Aya Kabbara, Veronique Paban, Mahmoud Hassan. The dynamic modular fingerprints of the human

brain at rest. NeuroImage, 2021, 227, pp.117674. 10.1016/j.neuroimage.2020.117674 . hal-03107402

\section{HAL Id: hal-03107402 \\ https://hal-amu.archives-ouvertes.fr/hal-03107402}

Submitted on 12 Jan 2021

HAL is a multi-disciplinary open access archive for the deposit and dissemination of scientific research documents, whether they are published or not. The documents may come from teaching and research institutions in France or abroad, or from public or private research centers.
L'archive ouverte pluridisciplinaire HAL, est destinée au dépôt et à la diffusion de documents scientifiques de niveau recherche, publiés ou non, émanant des établissements d'enseignement et de recherche français ou étrangers, des laboratoires publics ou privés. 


\title{
The dynamic modular fingerprints of the human brain at rest
}

\author{
A. Kabbara ${ }^{\text {a, }}$ V. Paban ${ }^{\text {b }}$, M. Hassan ${ }^{\text {a,c,* }}$ \\ a Univ Rennes, LTSI - U1099, Rennes F-35000, France \\ ${ }^{\mathrm{b}}$ Aix Marseille University, CNRS, LNSC, Marseille, France \\ ${ }^{\mathrm{c}}$ NeuroKyma, Rennes F-35000, France
}

\section{A B S T R A C T}

The human brain is a dynamic modular network that can be decomposed into a set of modules, and its activity changes continually over time. At rest, several brain networks, known as Resting-State Networks (RSNs), emerge and cross-communicate even at sub-second temporal scale. Here, we seek to decipher the fast reshaping in spontaneous brain modularity and its relationships with RSNs. We use Electro/Magneto-Encephalography (EEG/MEG) to track the dynamics of modular brain networks, in three independent datasets $(N=568)$ of healthy subjects at rest. We show the presence of strikingly consistent RSNs, and a splitting phenomenon of some of these networks, especially the default mode network, visual, temporal and dorsal attentional networks. We also demonstrate that between-subjects variability in mental imagery is associated with the temporal characteristics of specific modules, particularly the visual network. Taken together, our findings show that large-scale electrophysiological networks have modularity-dependent dynamic fingerprints at rest.

\section{Introduction}

Spontaneous brain activity changes continuously, over multiple temporal scales ranging from sub-second to years. Those fluctuations involve a set of networks known as resting-state networks (RSNs) (Damoiseaux et al., 2012; Raichle et al., 2001) . To decipher the ultra-fast dynamic reconfiguration of these RSNs and their crosscommunications, several functional studies have been conducted. Some studies have described the dynamic topological changes of functional networks using graph theoretical analysis (de Pasquale et al., 2015; Jiao et al., 2018; Kabbara et al., 2017). Others focused on detecting 'brain network states' fluctuating over time (E.A. Allen et al., 2014; Baker et al., 2014). The main idea is that spontaneous brain activity can be explained by a set of spatiotemporal network patterns. Most studies have been performed in combination with dimensionality reduction algorithms (such as K-means clustering (E.A. Allen et al., 2014), principal component analysis (Preti and Van De Ville, 2016; Leonardi et al., 2013), orthogonal connectivity factorization (Hyvärinen et al., 2016)), or model-based approaches, such as Hidden Markov Models (Baker et al., 2014). Features derived from these fast-dynamic analyses were also shown as potential neuromarkers for some brain diseases (Filippi et al., 2019; A Kabbara et al., 2018; Liu et al., 2019) and behavioral characteristics (Kenett et al., 2020; Tompson et al., 2018).

Emerging evidence shows that the human brain is a modular network partitioned into (modules') also called communities or clusters) denoting brain regions that are highly intra-connected and weakly connected with others (Bassett and Sporns, 2017). The modular organization of the human brain network and its dynamics were shown to be associated with aging (Meunier et al., 2009) and several task-related brain functions such as learning (Bassett et al., 2011) and cognitive efforts (Kitzbichler et al., 2011). While several resting-state functional magnetic resonance imaging (fMRI) studies have been conducted to investigate the time-dependence of brain modular networks (E.A. Allen et al., 2014; Jones et al., 2012; Zalesky et al., 2014), the evidence for rapid reshaping in spontaneous modular brain networks and their relationships with RSNs at timescales associated with fast cognition is very limited. To precisely track network dynamics, we need a modality that can match the rapid timescales of the underlying brain functions. In this context, electro/magneto-encephalography (EEG/MEG) can be used to describe those fast (sub-millisecond) modularity-dependent fluctuations.

Here, we hypothesized that the dynamic modular reorganization of the human brain at rest is characterized by a continuous process of separation and merging within and across different RSNs over time. To detect "modular brain states", we use a recently developed framework that allows to precisely quantify the fluctuations of the time-varying 'states' (A. Kabbara et al., 2019). Unlike other clustering algorithms, this framework detects the fast-transient changes in the brain modular structure and was shown to outperform other existing clustering algorithms in terms of spatiotemporal precision. We tested our hypothesis on three independent EEG/MEG datasets $(N=568)$ for healthy subjects at rest, source-reconstructed to 68 regions across the entire cortex. Dynamic brain networks were reconstructed using the EEG/MEG source connectivity technique using both power- and phase- couplings (Hassan and Wendling, 2018), combined with a sliding window approach and an algorithm to detect modular states (Fig. 1). Notably, our results revealed the presence of consistent network patterns for most participants and a splitting phenomenon of some of these networks, such as the default

\footnotetext{
* Corresponding author.

E-mail address: mahmoud.hassan@univ-rennes1.fr (M. Hassan).
} 

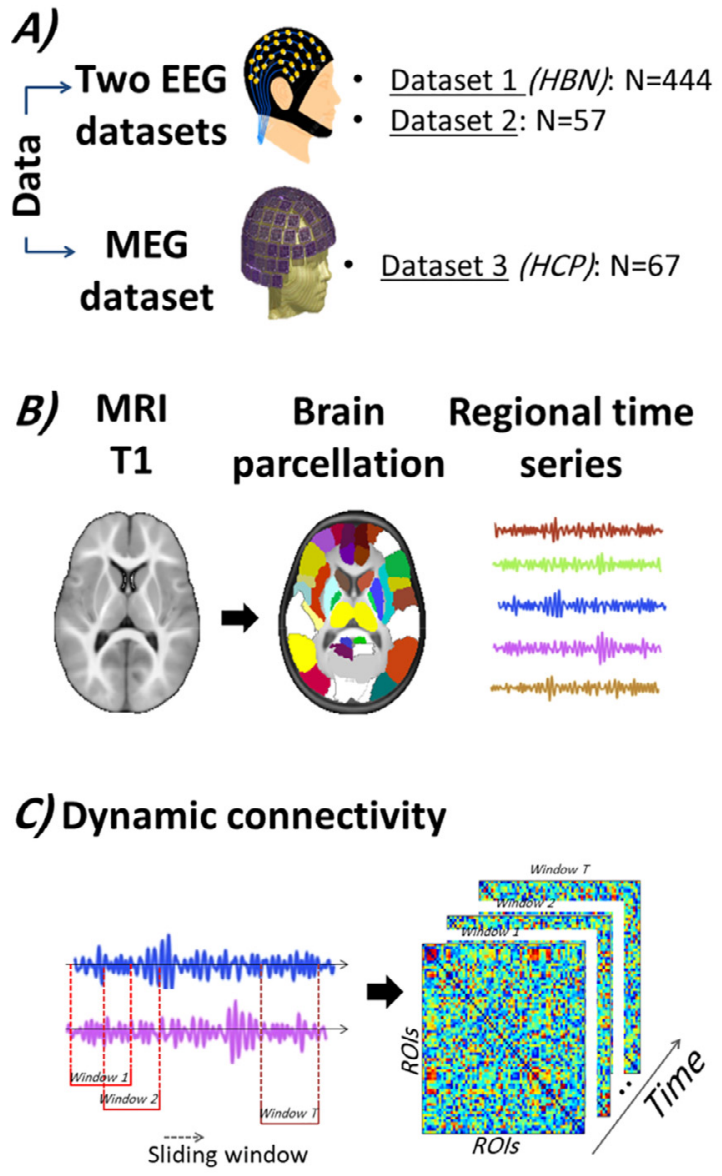

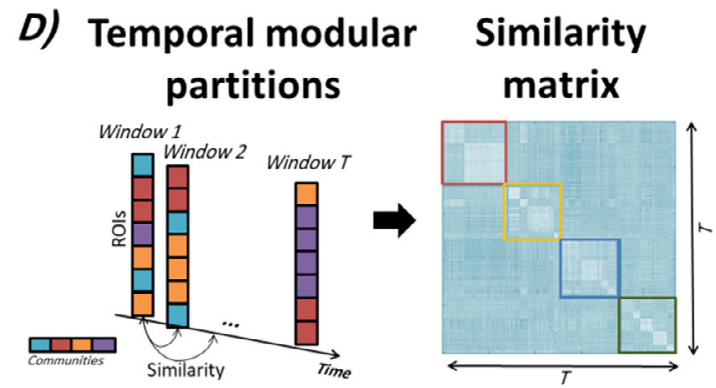

E) Modular states

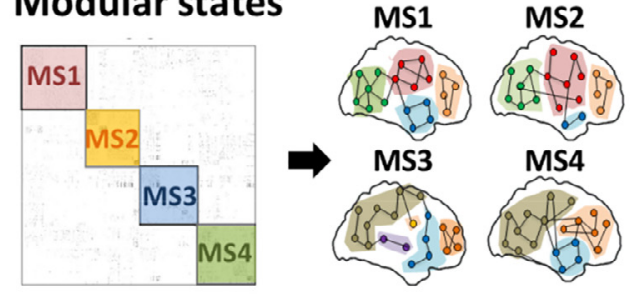

F) Modules related to RSNs

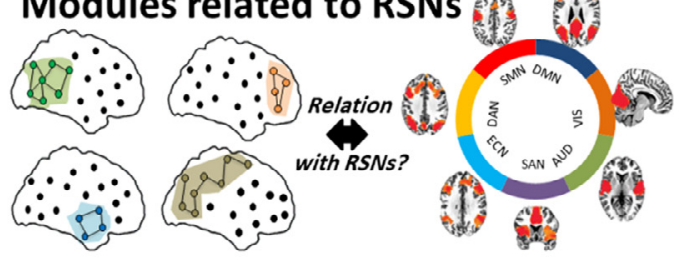

G) Temporal features

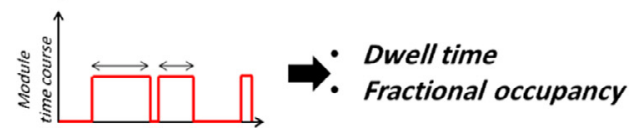

Fig. 1. Study pipeline. (A) Three datasets were analyzed: 1) Resting-state EEG data provided from the healthy brain network biobank including 444 subjects; 2) Resting-state EEG data acquired from 56 healthy subjects; and 3) Resting-state MEG data provided from the human connectome project including 61 subjects. (B) The template MRI was segmented into regions of interest (ROIs) by the means of an anatomical atlas (Desikan et al., 2006). Then, the regional time series of each subject were reconstructed using the weighted minimum norm estimate inverse solution (WMNE) for Datasets 1 and 2, and beamforming for Dataset 3. (C) Using a sliding window technique, the dynamic brain networks were computed. (D) The first step in the modularity-based algorithm was to parcellate each temporal network into communities. Then, the similarity between the temporal modular structures was assessed. (E) The similarity matrix was segmented into different communities where each one represents a modular state of specific spatial topology combining different time windows. (F) Following this, all the modules derived from the different MSs were extracted for each subject. Among them, we only retained those that presented strong association with RSNs (more than $80 \%$ of nodal overlap). Please see Supplementary materials for the definition of RSNs. (G) The mean dwell time and fractional occupancies were calculated for the main modules related to RSNs. (For interpretation of the references to color in this figure legend, the reader is referred to the web version of this article.)

mode, visual, temporal and dorsal attentional networks. We speculate that tracking the fast modular architecture of ongoing neuronal activity provides new insights into the dynamics of the large-scale electrophysiological network organization of the human brain.

\section{Results}

We performed our analysis on three independent datasets: 1) Resting-state EEG data provided from the Healthy brain network biobank comprising 444 subjects; 2) Resting-state EEG data acquired from 56 healthy subjects; and 3) Resting state MEG data provided from the Human Connectome Project including 61 subjects. The dynamic functional connectivity networks were assessed for each subject using the EEG/MEG source connectivity method (Hassan and Wendling, 2018). For EEG datasets (datasets 1 and 2), we used the weighted Minimum Norm Estimate (wMNE) followed by phasecouplings as recommended by previous EEG studies (Hassan et al., 2016; M. 2014). For the MEG dataset (dataset 3), we used the beamforming approach followed by envelope-couplings, as recommended in previous MEG studies (O'Neill et al., 2017a; Tijms et al., 2013), with correction for spatial leakage to reduce volume conduction effects. Then, a sliding window technique was applied forming a continuous series of snapshots characterizing the evolution of each individual's functional brain network (see Materials and Methods for details about the construction of EEG/MEG functional networks). Then, we applied the modularitybased algorithm that uses as an input the tensor of dynamic networks and produce modular states (MSs) fluctuating over time, where each MS represents a unique modular topology. Briefly, the algorithm detects the modular structures sharing the same topology by quantifying the similarity between all the computed temporal partitions. We then identified the individual modules presenting a strong association with one or several RSNs. The full pipeline of the study is illustrated in Fig. 1.

\subsection{6 states were identified for the first database}

Fig. 2 illustrates the 16 modules derived from the 444 subjects in the alpha band. It also reports the percentage of subjects exhibiting each of the modules. One can realize that three derived modules are related to the DMN: POST-DMN including the posterior components of the DMN 
DMN

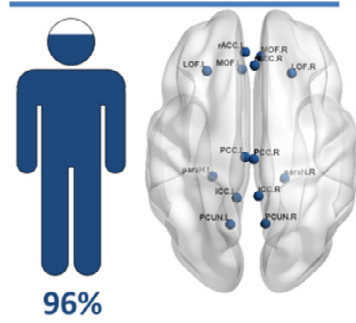

POST - DMN

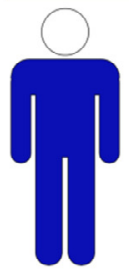

$88 \%$

VIS

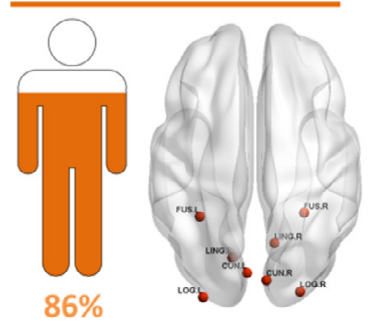

ANT - DMN

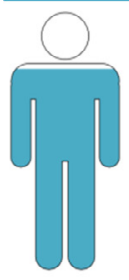

$86 \%$

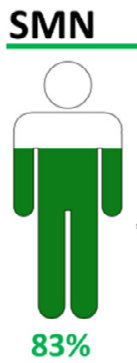

DAN

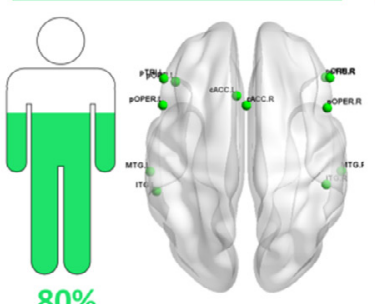

\section{L-TEMP}

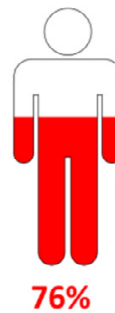

TEMP

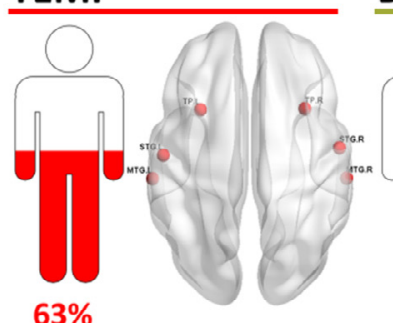

SAN

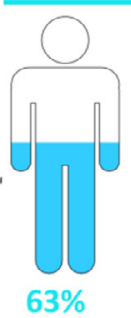

AUD+VIS

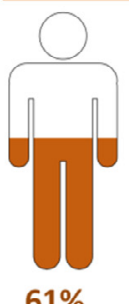

DMN + FPN

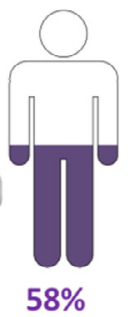

DAN + VIS

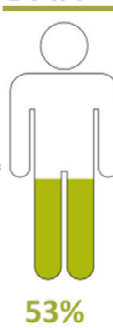

R-TEMP

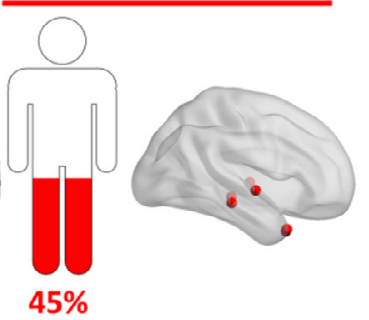

FPN

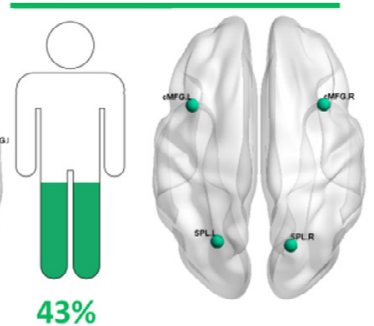

AUD + VIS + DAN

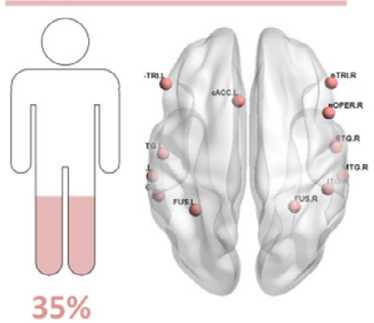

Ventral VIS

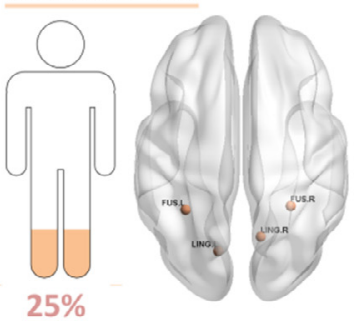

Fig. 2. Results of Dataset 1 obtained in the alpha band: Derived modules associated to RSNs and their corresponding percentage of subjects.

system (posterior cingulate, parahippocampal, precuneus, and inferior parietal lobule regions), ANT-DMN including the anterior components of the DMN (prefrontal regions), and DMN which represents the large module integrating both posterior and anterior parts into the same module. Also, three temporal modular configurations are depicted: L-TEMP and R-TEMP representing the left and right superior and inferior temporal regions, respectively; and TEMP that combines both left and right temporal modules. In addition, two modules related to the visual network were observed: the ventral-VIS, including the ventral regions of the visual network; and VIS integrating ventral and dorsal visual regions. Overall, the modules ranked from the highest to the lowest percentage of subjects are: DMN (present in 96\% of subjects), POST-DMN (present in $88 \%$ of subjects), VIS- visual network (present in $86 \%$ of subjects), ANT-DMN (present in $86 \%$ of subjects), SMN- somatomotor network (present in $83 \%$ of subjects), DAN- dorsal attentional network (present in $80 \%$ of subjects), L-TEMP (present in $76 \%$ of subjects), TEMP (present in $63 \%$ of subjects), SAN-salience network (present in $63 \%$ of subjects), AUD+VIS- (a module that combines both auditory and visual networks, present in $61 \%$ of subjects), DMN+FPN - (a module that combines both default mode and frontoparietal networks, present in $58 \%$ of subjects), DAN+VIS- (a module that combines DAN and VIS, present in $53 \%$ of subjects), R-TEMP (present in $45 \%$ of subjects), FPN (present in $43 \%$ of subjects), AUD+VIS+DAN- (a module combining AUD, VIS and DAN networks; present in $35 \%$ of subjects) and the ventral-VIS including lingual and fusiform visual regions (present in $25 \%$ of subjects). Results were consistent among several threshold values of functional connectivity matrices (see Table S1) and also within the beta frequency band (see Table S4).

\subsection{2 states were identified for the second database}

According to the second dataset (Fig. 3), 12 modules are extracted from the 57 subjects in the alpha frequency band. These modules are: POST-DMN (present in $98 \%$ of subjects), VIS (present in $94 \%$ of subjects), DAN (present in $91 \%$ of subjects), DMN (present in $84 \%$ of subjects), L-TEMP (present in $82 \%$ of subjects), ANT-DMN (present in $81 \%$ of subjects), SMN (present in $73 \%$ of subjects), AUD+VIS (present in $68 \%$ of subjects), DAN+VIS (present in $60 \%$ of subjects), TEMP (present in $45 \%$ of subjects), DMN+CCN (a module that combines DMN with cognitive control components, present in $32 \%$ of subjects) and SAN (present in $22 \%$ of subjects). Results were also consistent among several threshold values of functional connectivity matrices (see Table S2) and within the beta frequency band (see Table S5). 

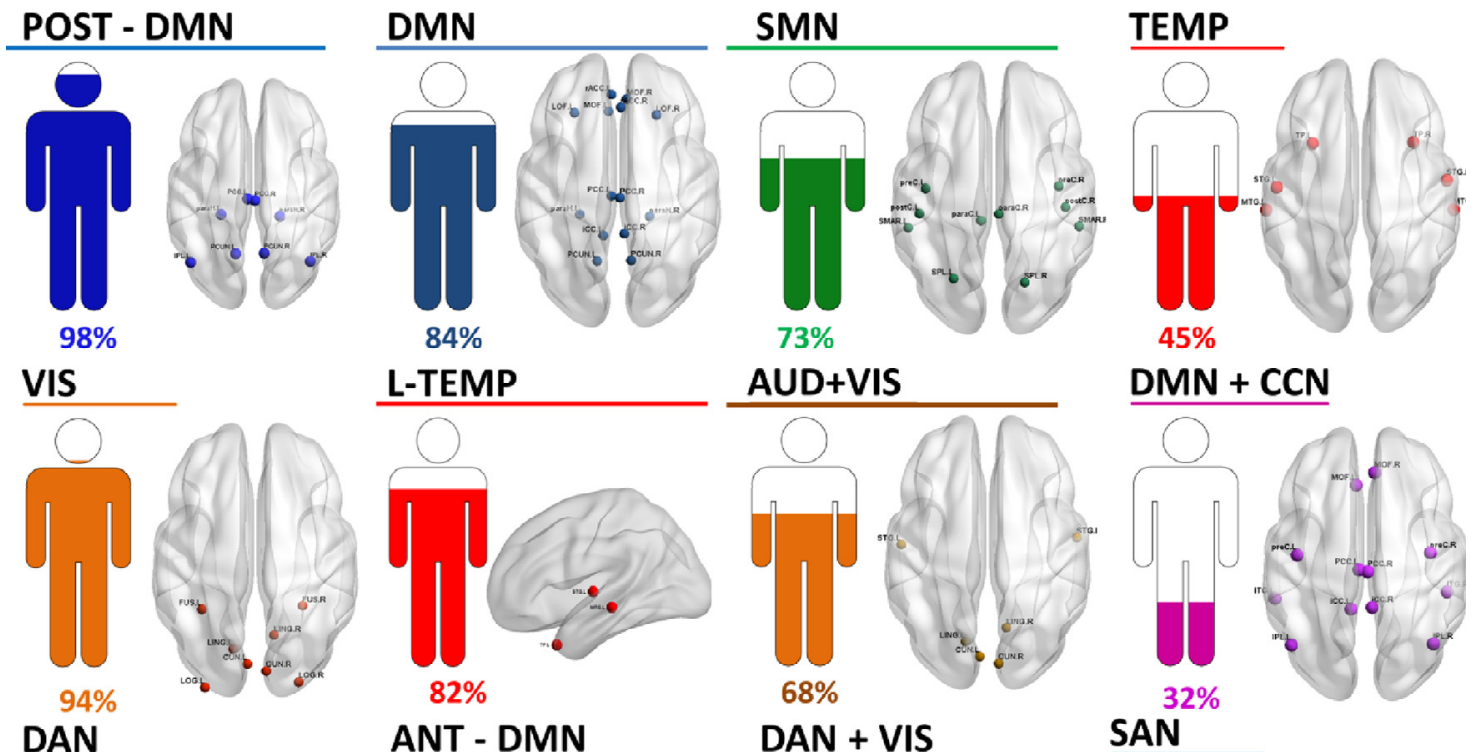

AUD+VIS

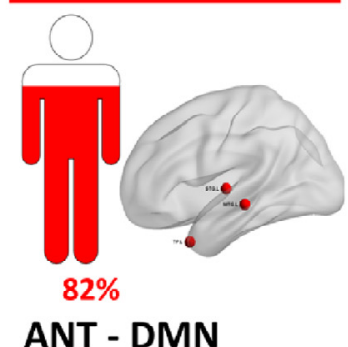

$$
68 \%
$$

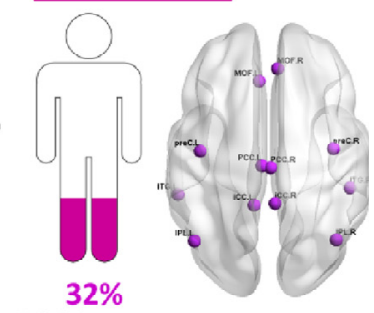

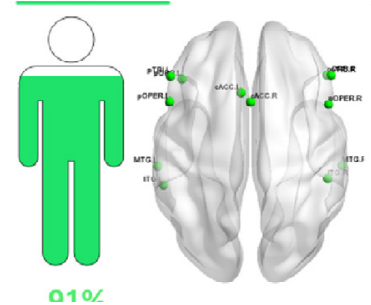

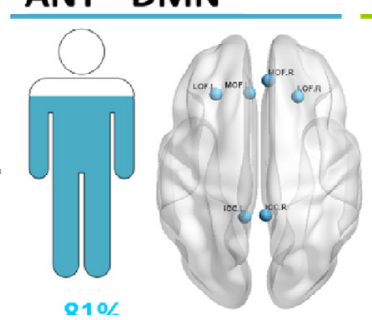

\section{DAN + VIS}

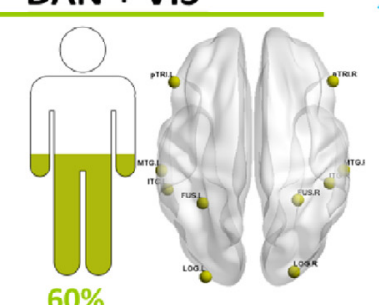

SAN

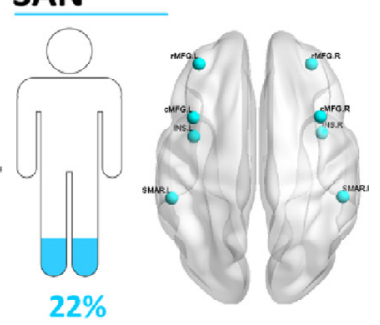

Fig. 3. Results of Dataset 2 obtained in the alpha band: Derived modules associated to RSNs and their corresponding percentage of subjects.

\subsection{0 states were identified for the third database}

In Fig. 4, we illustrate the results obtained in the alpha band for the third dataset showing 10 modules derived from the 61 subjects: DMN (present in $100 \%$ of subjects), POST-DMN (present in $95 \%$ of subjects), VIS (present in $88 \%$ of subjects), L-TEMP (present in $78 \%$ of subjects), SAN (present in $72 \%$ of subjects), DAN (present in $79 \%$ of subjects), SMN (present in $62 \%$ of subjects), AUD+VIS (present in $49 \%$ of subjects), DAN+VIS (present in 43\% of subjects) and CCN (present in 18\% of subjects). Results were consistent among several threshold values of functional connectivity matrices (see Table S3) and within the beta frequency band (see Table S6).

In summary, results obtained for the three datasets revealed fluctuating modules concordant with the well-known RSNs. In particular, the default mode network was the most consistent network among subjects in all datasets (reflected by the highest percentage of presence over subjects). Results also showed that some RSNs present various modular topologies over time such as DMN, temporal and the visual networks. In addition, modules that combine several RSNs are observed over time, reflecting cross-network interactions, as discussed below in more details.

Fig. 5 presents a step-by-step example of results obtained for a typical subject, where 8 MSs are derived. The similarities between the 30 modules extracted from all MSs and RSNs templates are assessed (Fig. 5.C). Among the 30 modules, 18 modules have survived the $80 \%$ threshold on the nodal overlap. These 18 modules are associated to 11 RSNs: DMN, SAN, SMN, VIS, LTEMP, DAN+VIS, AUD+VIS, POST-DMN, DMN+FPN, FPN, ANT-DMN. Visual inspection of the similarity matrix of Fig. 5.C reveals that DMN, SMN, VIS, ANT-DMN, AUD+VIS and LTEMP were representative of two or more modules while SAN, DAN+VIS and POSTDMN were associated to a single module. Fig. 5.D presents the dynamic fluctuations of the modules identified, where each module is color-coded according to its corresponding RSN.

\subsection{Dwell time and the fractional occupancy}

In order to quantify the temporal characteristics of each module, two metrics were computed: dwell time (DT), i.e. the average number of consecutive windows spent in a module; and the fractional occupancy (FO), reflecting the proportion of time spent in each module. As an example, the modules obtained in Fig. 5 ordered in terms of $\mathrm{FO}$ are: $\mathrm{DMN}(\mathrm{FO}=56 \%)$, VIS $(\mathrm{FO}=43 \%), \mathrm{DAN}+\mathrm{VIS}(\mathrm{FO}=43 \%)$, SMN $(\mathrm{FO}=31 \%)$, SAN $(\mathrm{FO}=19 \%)$, LTEMP $(\mathrm{FO}=14 \%)$, AUD+VIS $(\mathrm{FO}=$ $10 \%), \operatorname{ANT}-\mathrm{DMN}(\mathrm{FO}=4 \%), \operatorname{POSTDMN}(\mathrm{FO}=3 \%), \mathrm{DMN}+\mathrm{FPN}(\mathrm{FO}=3 \%)$, FPN $(\mathrm{FO}=3 \%)$. In terms of DT, the modules are ordered as follow: DMN (DT $=16 \%)$, VIS (DT $=10 \%)$, DAN+VIS (DT $=7 \%)$, SMN (DT=7\%), AUD + VIS $(\mathrm{DT}=7 \%)$, SAN (DT $=6 \%)$, POSTDMN(DT=6\%), LTEMP (DT=6\%), FPN (DT=5\%), DMN+FPN (DT=5\%).

Fig. 6 reports the FOs and the mean DTs of the modules obtained for each dataset. The DMN (or one of its modular configurations) has clearly the highest FO and DT over all datasets. The VIS network is shown as significant in terms of FO in dataset2. According to the DT, SMN and SAN are depicted as significantly stable modules in dataset 1 .

In summary, results obtained from all datasets points at the importance (and stability) of DMN and its role as a functional core network during rest, as detailed in Discussion.

\subsection{Correlation between the derived modules and mental imagery}

Finally, we seek at understanding if there is any correlation between the derived modules and the subject internal thoughts experienced during resting-state acquisition measured by the Resting-State Questionnaire (rsQ). Only such data was available for dataset 2 . More specifically, the five main indices derived from the rsQ (i.e. visual mental imagery, inner language, somatosensory awareness, inner musical experience, and mental manipulation of numbers) were correlated with 


\section{DMN}

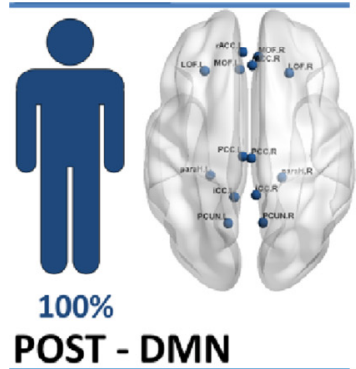

POST - DMN

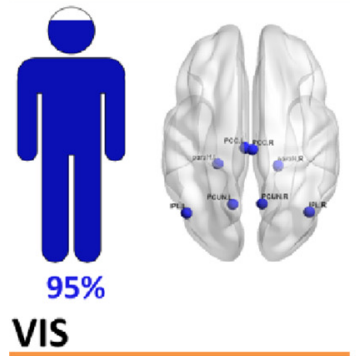

VIS

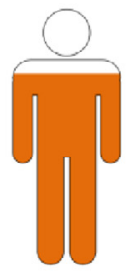

88\%

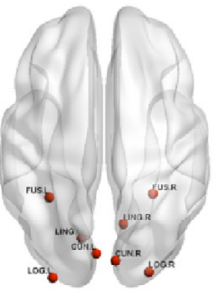

L-TEMP

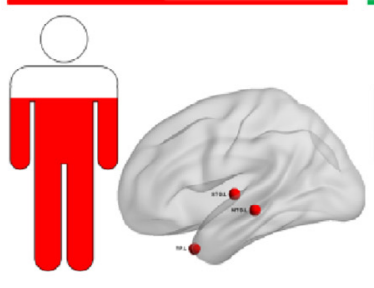

$78 \%$

SAN
SMN

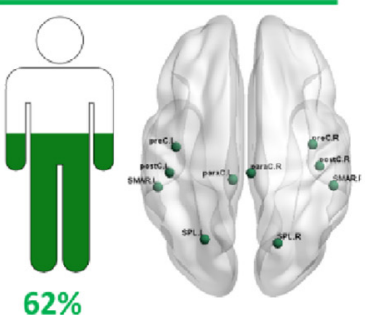

AUD+VIS

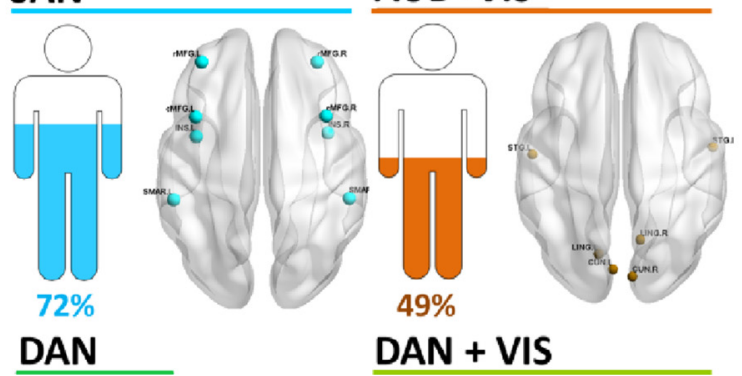

DAN

DAN + VIS

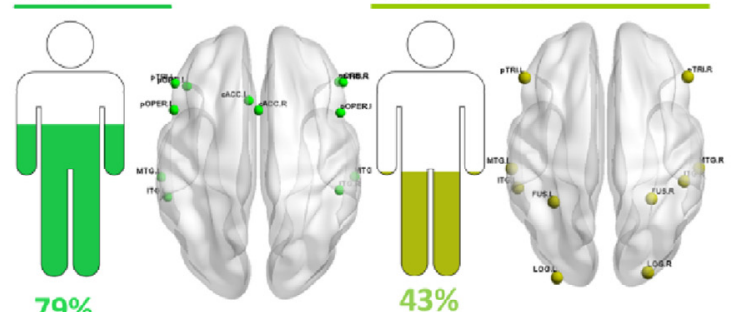

CCN

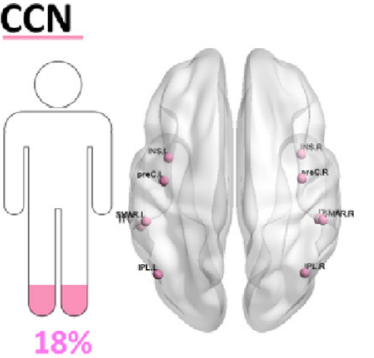

$18 \%$

$79 \%$

43\%

Fig. 4. Results of Dataset 3 obtained in the alpha band: Derived modules associated to RSNs and their corresponding percentage of subjects.

the temporal features of each module. No significant correlations were obtained between any of the rsQ scores and the DT of extracted modules.

Fig. 7 reports significant positive correlations between visual mental imagery and the fractional occupancy of VIS ( $p_{\text {Bonferroni corrected }}=$ $0.01 ; R=0.47), \quad$ DAN $\quad\left(p_{\text {Bonferroni corrected }}=0.006 ; R=0.491\right) \quad$ and AUD+VIS ( $p_{\text {Bonferroni corrected }}=0.0003 ; R=0.57$ ) obtained in the alpha band. In the beta band, results show positive correlations between visual imagery and the FO of AUD+VIS and DAN (see Figure S3). Results were also consistent across other threshold values (see Figure S2).

In summary, these results showed that individual variability in the visual imagery experienced during acquisition was positively related to the occupancy of specific modules, mainly VIS, DAN and VIS+AUD.

\section{Discussion}

Here, we have shown how fast changes in the modular architecture of large-scale electrophysiological networks shape spontaneous brain activity. We used a recently developed algorithm that extracts repetitive modular brain states alternating over time. As opposed to traditional approaches, the distinctive feature of the applied method resides in tracking modular variations of brain networks. The framework was applied on three independent EEG/MEG datasets, and revealed that RSNs experience continuous modular changes reflected by a process of separation and merging within- and between- the resting networks.

In particular, DMN switches dynamically its modular topology, in line with many previous studies suggesting that the DMN can actually be decomposed into subcomponents, mainly anterior and posterior (Andrews-Hanna et al., 2007; Moussa et al., 2011; Wens et al., 2019). The process of association and dissociation within DMN components was also revealed by Allen et al. (E.A. Allen et al., 2014), where brain states were described using K-means clustering. More importantly, sev- eral studies have also showed that the dynamic states transition leads to the inclusion of some FPN regions in the DMN in some brain states (E.A. Allen et al., 2014; Liu et al., 2019), which was also obtained in our study (results of dataset 1). Similarly, the temporal network alternates its reconfiguration between left, right and complete modules. This finding is in line with previous results depicting the left part of the temporal network as an independent network state (Baker et al., 2014). The dynamic modular behavior of the resting brain was also revealed by the occurrence of modules integrating different RSNs. For instance, the DAN expands dynamically its network to include visual components. The dynamic inclusion of these networks reflects the presence of a high correlation between them, which was supported in previous studies (E.A. Allen et al., 2014; Liu et al., 2019).

Our findings agree with previous studies suggesting that dynamic changes in brain networks are present during spontaneous activity. As an example, (Zalesky et al., 2014) showed that some brain regions are transmodal (i.e., connected to different resting state networks over time). These regions are highly dynamic and change their modular affiliation over time. A similar observation was obtained in a previous study where hubs dynamically alternate its role between provincial and connector (Kabbara et al., 2017). This dynamic process of splitting and merging the different sub-systems during time allows the brain to balance segregated and integrated neural dynamics. To test whether there may be a sort of hierarchy in terms of the consistency of brain regions, we also reported, for each RSN, the regions included and their contributions across subjects of all datasets (in\%). Once a module was associated to a specific RSN, the overlapping nodes were identified to ultimately compute the rate of their inclusion across all subjects and datasets. Figure $\mathrm{S} 1$ shows that despite the inter-subject variability, some specific brain regions contribute more consistently to a specific RSN than others.

The DMN (in its different configurations) was the most consistent module obtained across subjects, since it had the highest percentage of 


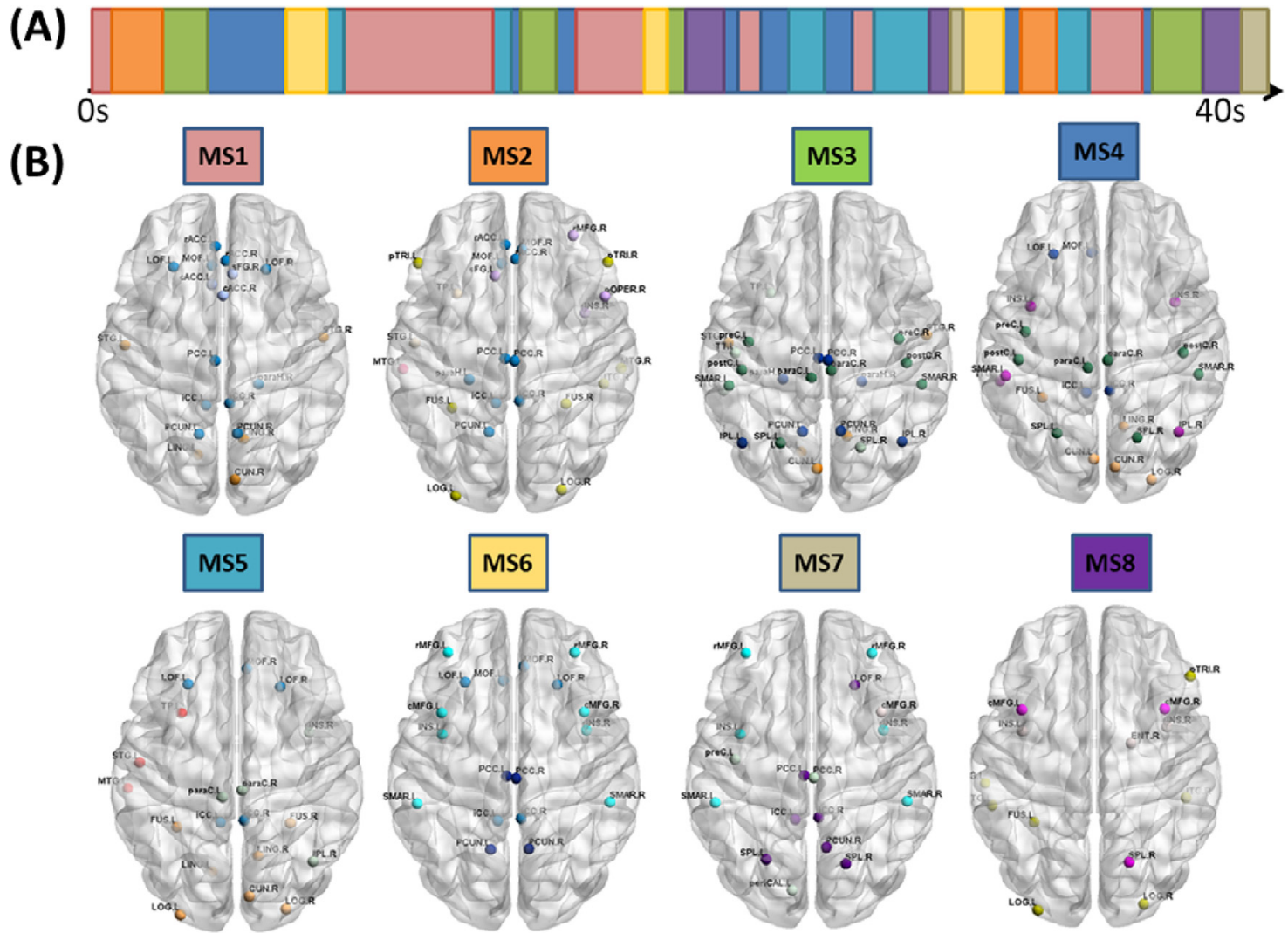

(C)

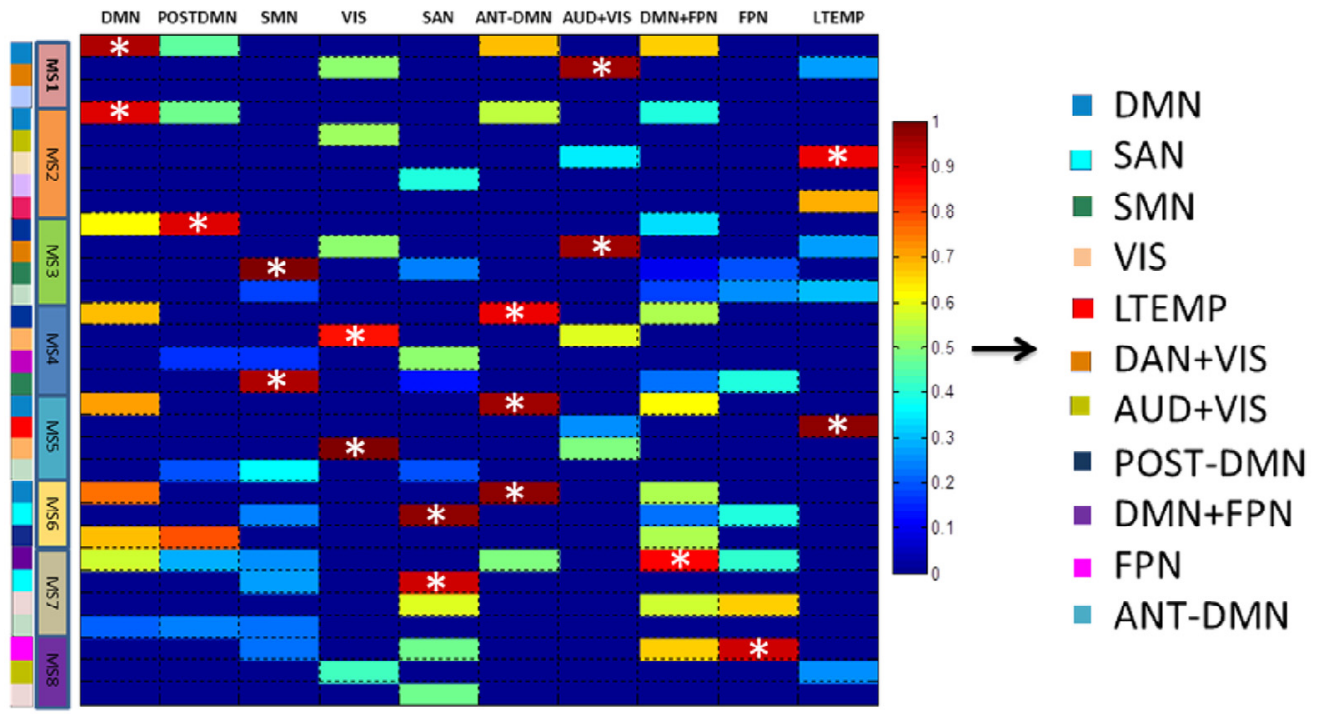

(D)

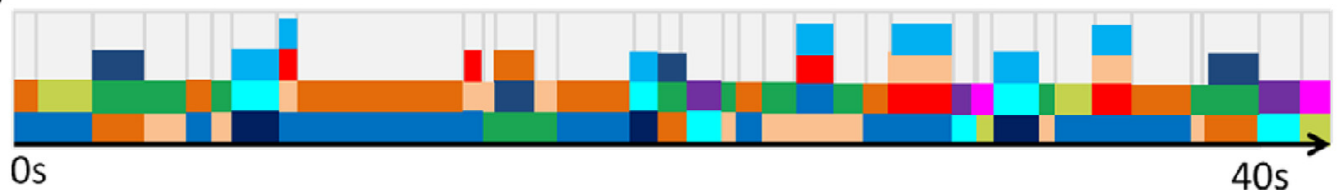

Fig. 5. Results of a typical subject. (A) dynamic fluctuation of the 8 modular states extracted for this specific subject, (B) spatial representation of all modules derived from each MS. (C) similarity matrix between all MSs modules and RSN templates (for simplicity, only RSNs showing an overlap greater than $80 \%$ with one of the modules were mentioned), * marks the overlap values higher than $80 \%$. (D) dynamic fluctuations of modules surviving the $80 \%$ overlap threshold and associated to RSN templates. These modules are color-coded according to the corresponding RSNs (shown on the right of Fig. 5.C). 
(A)
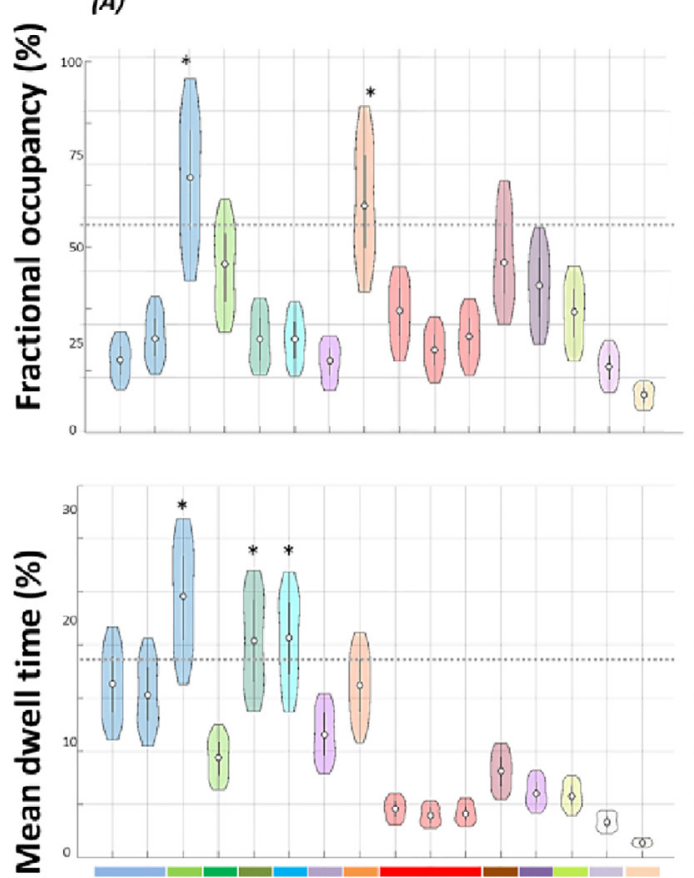

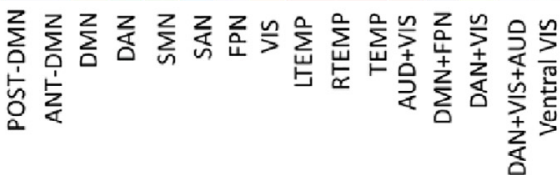

(B)

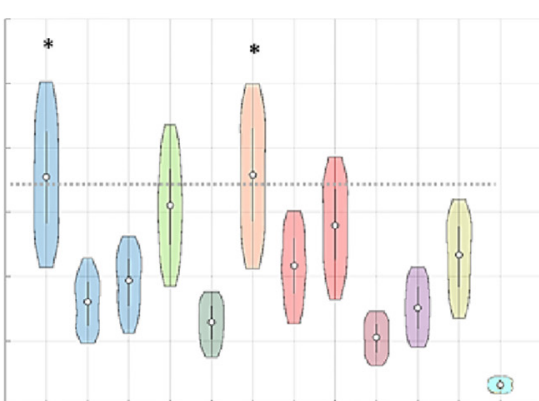

(c)
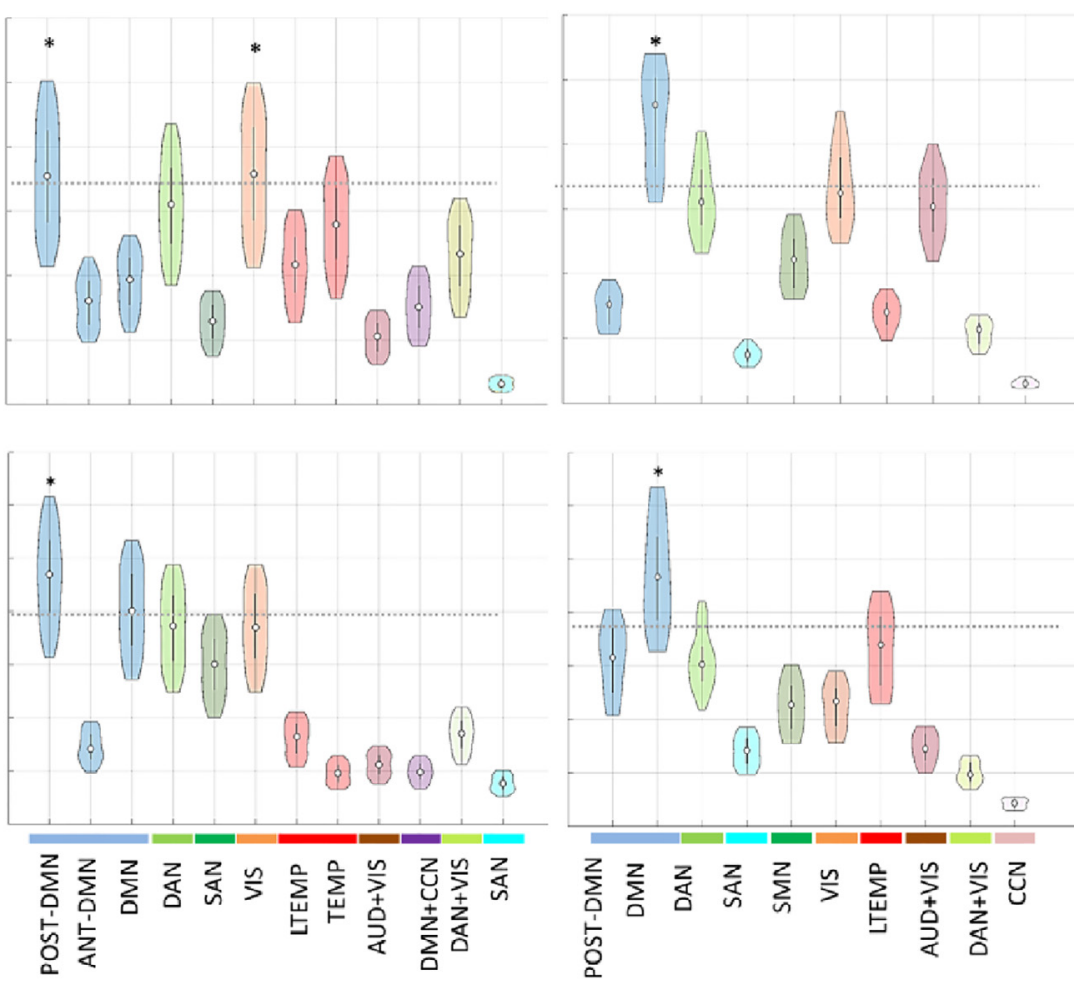

Fig. 6. Violin plots showing fractional occupancy and mean dwell time of derived modules obtained for (A) Dataset1, (B) Dataset2 and (C) Dataset3. The horizontal dashed line that appears in each plot denotes the mean plus two standard deviations. ${ }^{*}$ mark significant modules (average $>$ mean value +2 standard deviations).
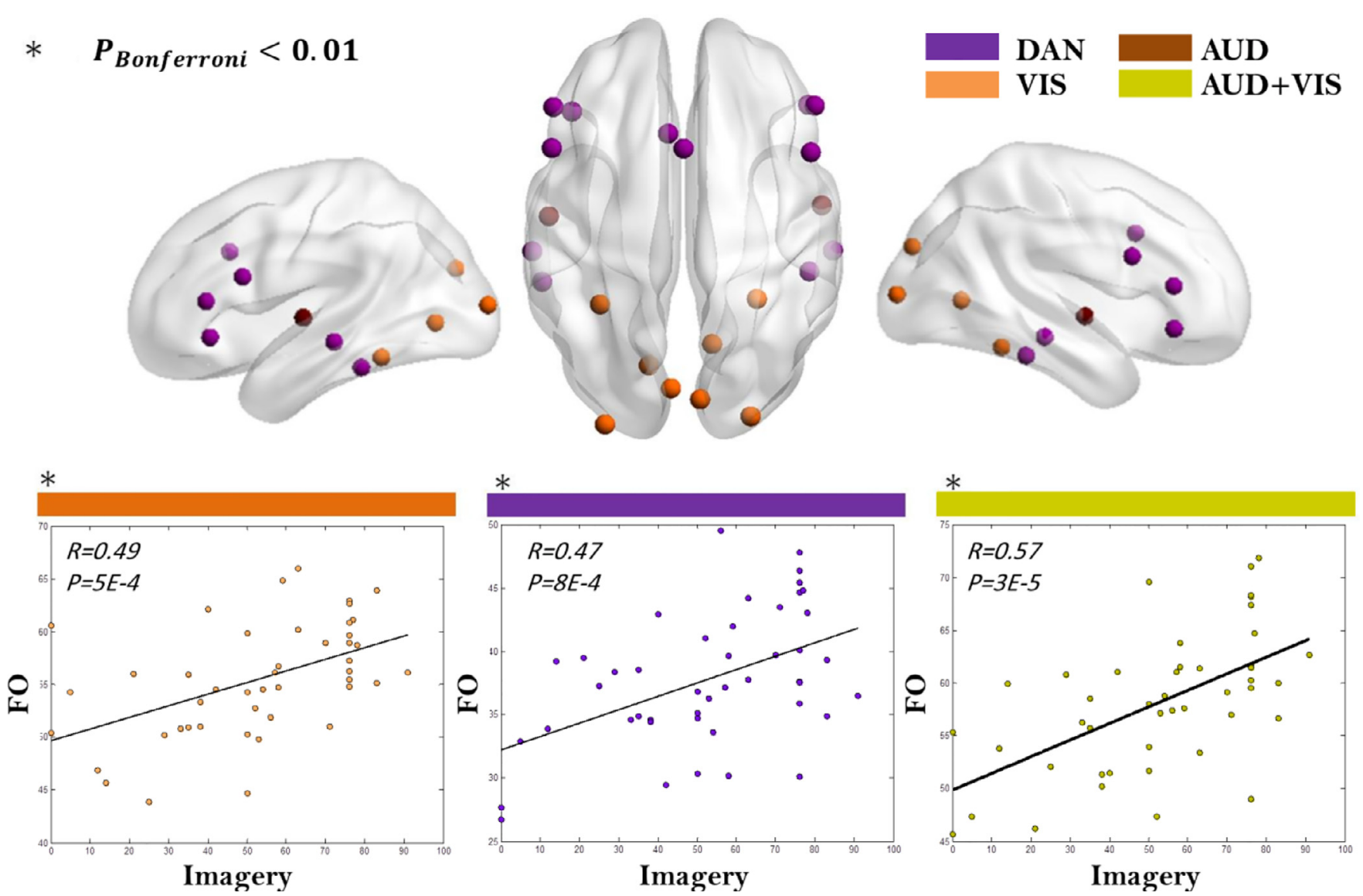

Fig. 7. Significant associations between visual mental imagery and fractional occupancies of VIS, AUD and DAN modules in the alpha band (dataset 2). 
presence over subjects/datasets, with the high fractional occupancy values. These findings highlight the key role of DMN in integrating information in spontaneous brain activity, in accordance with the presence of a large proportion of hubs associated to this network (de Pasquale et al., 2015; Kabbara et al., 2017; van den Heuvel and Sporns, 2013). Furthermore, DMN configurations had the greatest stability over time (reflected by the highest mean dwell duration). Such findings support previous studies showing that functional connections within the richclub core (where most regions are affiliated to the DMN) exhibit the greatest stability over time. In these studies, the high temporal stability of DMN has been associated with high dependency on the underlying structural brain topology, since high similarity was obtained between resting-state functional and structural networks when the sample duration increases. In contrast to this, modules with low dwell time were transient and exhibited greatest variability during time reflecting the dynamic functional coordination. In our study, the derived transient modules depend on each subject and database. Mostly, they belong to high-level cognitive networks, attentional networks and the sensory networks. In all databases, these transient modules showing significant lower dwell times are those integrating multiple RSNs (DAN+VIS, AUD+VIS...). One possible explanation is that individuals dynamically engage in several mental thoughts during resting periods, while imagery and mind-wandering remain the predominant activities (Delamillieure et al., 2010; Doucet et al., 2012). Thus, the perspective of looking at the brain as a dynamic system where stable activity is intertwined by transient functional variabilities is supported by many studies (Honey et al., 2007; Liu et al., 2019; Van De Ville et al., 2010).

In addition, our study highlights the significant presence of the visual network showing a high occupancy rate during time (results of datasets 1 and 2). Such observation can be associated with the dominance of the visual imagery activity exhibited by most subjects during resting state acquisition (Delamillieure et al., 2010). More interestingly, the individual variability in the visual imagery experienced during acquisition was revealed to be positively correlated to the fractional occupancy of VIS network (Fig. 7). Similar correlations were reported by previous studies (Pipinis et al., 2017; Stoffers et al., 2015). In addition, the significant relationships assessed between the mental imagery with AUD, DAN and VIS might explain the cross-interactions observed between these networks forming one large module over time (the presence of DAN+VIS+AUD observed in the results of dataset 1, Fig. 2).

Across the three datasets, our results showed 9 common RSNs: DMN, POST-DMN, VIS, SMN, SAN, LTEMP, VIS+AUD, DAN+VIS. These striking consistent results have been obtained independently from the technique used to record signals (EEG or MEG), preprocessing steps (automatic in dataset 1 vs. manual in dataset 2 and 3), source reconstruction (wMNE vs. Beamforming), adjacency matrices thresholding value, EEG/MEG frequency bands (alpha vs. beta), atlas parcellation (68 Desikan Killiany vs $78 \mathrm{AAL}$ ) and functional connectivity measures (phase vs. envelope couplings), as well as either with or without correcting the zero-lag correlations.

However, other modules arise from each dataset (Figs. 2,3,4). The inter-subject variability was also revealed by the percentage of subjects showing each derived module. Among the same dataset, these individual differences are thought to be associated with variability in cognitive and behavioral functions. This has been supported by different studies showing that the dynamic network characteristics significantly correlate with intelligence, creativity and executive function (Bassett et al., 2015; Kenett et al., 2020; Tompson et al., 2018). Here, between-subjects variation in the temporal characteristics of specific modules, mainly VIS, AUD and DAN, was associated with self-report rating of mental visual imagery as measured by the resting-state questionnaire. The dependence of observed brain activity on the inner thoughts and feeling experienced during resting acquisition was emphasized by multiple studies (Diaz et al., 2016; Pipinis et al., 2017; Stoffers et al., 2015).

Despite the overall consistency of our findings, results over the three datasets were not perfectly the same. For instance, the DMN were present $96 \%, 98 \%$ and $100 \%$ over dataset 1,2 and 3 respectively while the VIS network was present $86 \%, 94 \%$ and $88 \%$. Also results showed the absence of the ANT-DMN in dataset 3, the absence of FPN in dataset 2 and 3 and the absence of CCN in dataset1. The discrepancy of results obtained from different datasets may be related to some differences in the datasets such as the sample size, age of subjects and the conditions of experiments (i.e. eyes closed/eyes opened). In fact, while RSNs have been successfully extracted in both eyes-opened (E.A. Allen et al., 2014; Baker et al., 2014; de Pasquale et al., 2018) and eyes-closed conditions (Bernas et al., 2018; Kabbara et al., 2017; Owen et al., 2013), many studies have been conducted to investigate the functional connectivity differences in RSNs between eyes closed and eyes opened conditions (Agcaoglu et al., 2019; Patriat et al., 2013; Van Dijk et al., 2010; Yan et al., 2009). Our results on dataset 3 (acquired in eyes opened condition) showing the highest DMN occurrence (100\%) among other datasets (acquired in eyes closed condition) can be related with the studies revealing higher DMN functional connectivity in the eyes opened as compared to the eyes closed condition (Van Dijk et al., 2010; Yan et al., 2009). An additional cause for the discrepancy of results between datasets is the use of different modalities (MEG/EEG). More precisely, MEG/EEG differences proved to arise particularly when investigating transient resting-state functional connectivity patterns (Coquelet et al., 2020).

The relevance of the alpha to beta frequency range $(8-30 \mathrm{~Hz})$ in driving spontaneous large-scale neuronal interactions was revealed by multiple EEG/MEG studies (Brookes et al., 2011; de Pasquale et al., 2015; Hipp et al., 2012; Kabbara et al., 2017; Liu et al., 2010). Since correlation patterns depend on the underlying oscillation frequency (Brookes et al., 2011; Hipp et al., 2012; Vidaurre et al., 2018), we have verified the reproducibility of the obtained results in these two frequency bands. The main conclusions of the study remain intact (see Table S4, Table S5, Table S6, Figure S3): i) distinct modules concordant with the well-known RSNs fluctuate during time, ii) the default mode network is detected as the most consistent, dominant and stable module which dynamically alternates its modular topology, iii) modules that combine several RSNs are observed during time, reflecting cross-network interactions such as DAN-VIS, and iv) significant positive correlation was revealed between the fractional occurrences of some specific modules and the mental imagery. Nevertheless, slight differences were observed in the derived modules and their temporal characteristics between the two frequency bands.

From a methodological viewpoint, we have adopted in each dataset the pipeline (from data processing to networks construction) used by the previous studies dealing with the same datasets. Therefore, for EEG datasets, we used the wMNE/PLV combination to reconstruct dynamic networks, since it is supported by several studies on resting EEG and two comparative studies (Hassan et al., 2016; M. 2014). For the MEG dataset, beamforming construction combined with amplitude correlation (and orthogonalization) between band-limited power envelops was used by multiple studies using the MEG HCP data (Brookes et al., 2012; Colclough et al., 2015). The suitable window width is a crucial issue in reconstructing dynamic functional networks. On the one hand, short windows do not contain sufficient information to accurately estimate connectivity. On the other hand, large windows might fail to capture the temporal changes of brain networks. Hence, the ideal is to choose the shortest window that guarantees a sufficient number of data points over which connectivity is computed. This depends on the frequency band of interest that affects the degree of freedom in time series. It also depends on the correlation measure used. In EEG datasets, we adopted the recommendation of Lachaux et al. (Lachaux et al., 2000) in selecting the smallest appropriate window length offering 6 number of 'cycles' at the given frequency band. The reproducibility of resting state results whilst changing the size of the sliding window was validated in a previous study (Kabbara et al., 2017). In MEG, we used the same correlation method with the corresponding sliding window size $(0.5 \mathrm{~s})$ used in previous stud- 
ies dealing with the same dataset (Colclough et al., 2016a; O'Neill et al., 2017b).

While EEG/MEG modalities present a key advantage for tracking dynamic brain processes in the time frame in which these processes occur (namely in the sub-second range), they are limited in terms of spatial coverage. Basically, the main cause of this poor spatial resolution is the "volume conduction" problem which distorts signals, inducing, at each scalp position, a mixture effect of the underlying sources. Readers may refer to (Schoffelen and Gross, 2009) for more details about this issue. Although the EEG/MEG source connectivity method contributes to enhance the spatial resolution by reducing the effects of the volume conduction problem, it remains unable to completely remove source leakage effects (Hassan and Wendling, 2018). More precisely, it is difficult to pinpoint the activity originating from distinct but closely adjacent sources. For this reason, in our study, we avoided the use of a high number of ROIs. Thus, we used 68 anatomical ROIs in EEG datasets, and 78 ROIs in MEG dataset as done in previous resting-state studies showed that these ROIs are sufficient in extracting the global characteristics of the brain networks with acceptable spatial resolution while minimizing the problem of spurious connections between "very close sources" (Kabbara et al., 2017). The Desikan_Killiany atlas (68 ROIs) was also used to extract useful information focusing on investigating the "large-scale" networks derived from spontaneous activity in the context of personality traits (Aya Kabbara et al., 2019), and brain disorders (A. Kabbara et al., 2018). In addition, we have limited our source space to the cortex without including sub-cortical structures.

In this study, we used a proportional threshold (highest $15 \%$ of the edge's weights) to remove weak connections. The stability of networkbased features across proportional thresholds was indeed supported by (Garrison et al., 2015) in contrary to absolute thresholds. In addition, applying a proportional threshold is important to ensure equal density between networks derived from different time windows and subjects. Nevertheless, and in order to ensure that the obtained results are not sensitive to the threshold value, we performed our analysis across three proportional thresholds: $5 \%, 15 \%, 30 \%$. High agreement among the obtained results was found, see Supplementary Materials (Table S1, Table S2, Table S3, Figure S2).

To extract the fast transient modules, we have applied the modularity-based algorithm that extracts the main modular brain states fluctuating over time (A. Kabbara et al., 2019). Other strategies aiming at identifying the connectivity states exist such as K-means clustering, ICA and PCA for instance. However, in these frameworks, states are identified without considering the modular organization of networks. Instead, the algorithm used in the present study performs its segmentation by looking at the brain as a dynamic modular network. In a previous study, a quantitative comparison using simulated data was performed between the modularity-based algorithm, K-means clustering (E.A. Allen et al., 2014), independent component analysis (O'Neill et al., 2017b) and the consensus clustering (Rasero et al., 2017), and it was shown that the framework used here outperformed the other techniques in terms of spatial and temporal accuracy.

\section{Materials and methods}

\subsection{EEG datasets}

\subsubsection{Dataset 1 (HBN)}

4.1.1.1. Participants. As part of the Healthy Brain Network (HBN) Biobank release 1 (Alexander et al., 2017) http://fcon_1000.projects. nitrc.org/indi/cmi_healthy_brain_network/sharing_neuro.html, restingstate EEG data were collected from 444 healthy subjects (239 female). The release originally included 603 subjects, but data from 159 subjects were rejected after pre-processing and visual inspection. Subjects are healthy and aged between 5 and 21 years old. The Ids of the 444 participants are listed in Table S7 (see Supplementary materials)
4.1.1.2. Data acquisition and pre-processing. High-density EEG data are recorded in a sound-shielded room at a sampling rate of $500 \mathrm{~Hz}$ with a bandpass of 0.1 to $100 \mathrm{~Hz}$, using a 128-channel EEG geodesic hydrocel system by EGI. The recording reference is at $\mathrm{Cz}$ (vertex of the head). The impedance of each electrode is checked prior to recording, to ensure good contact, and is kept below $40 \mathrm{kOhm}$. Each EEG session consisted of $5 \mathrm{~min}$ resting period (in eyes-closed condition). As provided by the HBN, EEG signals were preprocessed using Automagic Matlab toolbox (Pedroni et al., 2019), visual inspection was also done on the data after automatic preprocessing. Briefly, it consists of interpolating the noisy, flat or outlier channels. The Multiple Artifact Rejection Algorithm (MARA) which automatizes the process of independent component analysis (ICA) was used to detect and reject artifacts such as the eye blinks and the movement artifacts (Winkler et al., 2011). Then, four artifact-free epochs of 40-s length were selected for each participant. This epoch length was used in a previous study, and was considered as a good compromise between the needed temporal resolution and the results reproducibility (Kabbara et al., 2017).

\subsubsection{Dataset 2}

4.1.2.1. Participants. A total of 56 healthy subjects were recruited ( 29 female). The mean age was 34.7 years old $(S D=9.1$ years, range $=18$ 55). Education ranged from 10 years of schooling to a $\mathrm{PhD}$ degree. None of the volunteers reported taking any medication or drugs, nor suffered from any past or present neurological or psychiatric disease. The study was approved by the "Comité de Protection des Personnes Sud Méditerranée" (agreement $\mathrm{n}^{\circ} 10-41$ ). Same data were used in previous studies ((Kabbara et al., 2020)Aya Kabbara et al., 2019; Paban et al., 2019). After EEG acquisition, all participants have completed the resting-state questionnaire (ReSQ). This latter consists of 62 items organized by five main types of mental activity: visual mental imagery, inner language, somatosensory awareness, inner musical experience, and mental manipulation of numbers (Delamillieure et al., 2010). Using a scale ranging from 0 to $100 \%$, each participant rated the percentage of time spent in each mental activity during the resting-state EEG acquisition, such that the total score for the five types of activities equaled $100 \%$.

4.1.2.2. Data acquisition and preprocessing. Each EEG session consisted in a 10-min resting period with the participant's eyes closed. Participants were seated in a dimly lit room, were instructed to close their eyes, and then to simply relax until they were informed that they could open their eyes. Participants were informed that the resting period would last approximately $10 \mathrm{~min}$. The eyes-closed resting EEG recordings protocol was chosen to minimize movement and sensory input effects on electrical brain activity. EEG data were collected using a 64-channel Biosemi ActiveTwo system (Biosemi Instruments, Amsterdam, The Netherlands) positioned according to the standard 10-20 system montage, one electrocardiogram, and two bilateral electro-oculogram electrodes (EOG) for horizontal movements. Nasion-inion and preauricular anatomical measurements were made to locate each individual's vertex site. Electrode impedances were kept below $20 \mathrm{kOhm}$. The pre-processing was addressed using the same preprocessing steps as described in several previous studies dealing with EEG resting-state data (A Kabbara et al., 2018, 2017). Briefly, bad channels (signals that are either completely flat or contaminated by movement artifacts) were identified by visual inspection, complemented by the power spectral density. These bad channels were then recovered using an interpolation procedure implemented in Brainstorm by using neighboring electrodes within a $5-\mathrm{cm}$ radius. Epochs with voltage fluctuations between $+80 \mu \mathrm{V}$ and $-80 \mu \mathrm{V}$ were kept. Four artifact-free epochs of 40-s length were selected for each participant.

\subsubsection{Dynamic brain networks construction}

For the two EEG datasets, dynamic brain networks were reconstructed using the "EEG source connectivity" method (Hassan and Wendling, 2018) combined with a sliding window approach as detailed 
in (A. Kabbara et al., 2018, 2017). "EEG source connectivity" involves two main steps: i) solving the inverse problem in order to estimate the cortical sources and reconstruct their temporal dynamics, and ii) measuring the functional connectivity between the reconstructed timeseries.

Briefly, the steps performed were the following:

1 EEGs and MRI template (ICBM152) were coregistered through the identification of anatomical landmarks by using Brainstorm (Tadel et al., 2011).

2 A realistic head model was built using the OpenMEEG (Gramfort et al., 2010) software.

3 A Desikan-Killiany atlas-based segmentation approach was used to parcellate the cortical surface into 68 regions (Desikan et al., 2006).

4 The weighted minimum norm estimate (wMNE) algorithm was used to estimate the regional time series (Hamalainen and Ilmoniemi, 1994).

5 The reconstructed regional time series were filtered in alpha $8-13 \mathrm{~Hz}$ and beta $13-30 \mathrm{~Hz}$ frequency bands, shown to be the most involved frequency bands at rest.

6 To compute the functional connectivity between the reconstructed regional time-series, we used the phase locking value (PLV) metric (Lachaux et al., 2000) defined by the following equation:

$$
P L V(t)=\mid \frac{1}{\delta} \int_{t-\delta / 2}^{t+\delta / 2} \exp \left(j\left(\varphi_{y}(t)-\varphi_{x}(t)\right) d \tau \mid\right.
$$

where $\varphi_{y}(t)$ and $\varphi_{x}(t)$ are the unwrapped phases of the signals $x$ and $y$ at time $t$. The Hilbert transform was used to compute the instantaneous phase of each signal. $\delta$ denotes the size of the window in which PLV is calculated. Dynamic functional connectivity matrices were computed for each epoch using a sliding window technique (A. Kabbara et al., 2018 , 2017). It consists in moving a time window of certain duration $\delta$ along the time dimension of the epoch, and then PLV is calculated within each window. As recommended by (Lachaux et al., 2000), the number of cycles should be sufficient to estimate PLV in a compromise between a good temporal resolution and a good accuracy. The smallest number of cycles recommended equals to 6 . For instance, in the alpha band, we chose the smallest window length of $571 \mathrm{~ms}$ that is equal to $\frac{6}{\text { central frequency }}$.

1 To ensure equal network density for all the dynamic networks computed across time, a proportional (density-based) threshold was applied in a way to keep the top $15 \%$ of connectivity values in each network.

\section{2. $M E G$ dataset (HCP)}

\subsubsection{Participants}

As part of the HCP MEG2 release (Van Essen et al., 2012), restingstate MEG recordings were collected from 61 healthy subjects (38 women). The release included 67 subjects, but six subjects were omitted from the analysis as their recordings failed to pass the quality control checks (including tests for excessive SQUID jumps, sensible power spectra, correlations between sensors, and availability of sufficient good quality recording channels). All subjects are young (22-35 years of age) and healthy.

\subsection{2. $M E G$ recordings and pre-processing}

The acquisition was performed using a whole-head Magnes 3600 scanner (4D Neuroimaging, San Diego, CA, USA). Resting state measurements were taken in three consecutive sessions of 6 min each. During the scan the subject is instructed to relax with eyes open and maintain fixation on a projected crosshair presented on a dark background. Data were provided pre-processed, after passing through a pipeline that removed artefactual segments, identified faulty recording channels, and regressed out artefacts which appear as independent components in an ICA decomposition with clear artefactual temporal signatures (such as eye blinks or cardiac interference).

\subsubsection{Dynamic brain networks construction}

Here, we adopted the same pipeline used by the previous studies dealing with the same dataset (Colclough et al., 2015). Thus, to solve the inverse problem, we have applied a linearly constrained minimum variance beamformer (Van Veen et al., 1997). Pre-computed singleshell source models are provided by the HCP and the data covariance were computed separately in the $1-30 \mathrm{~Hz}$ and $30-48 \mathrm{~Hz}$ bands as in (Colclough et al., 2016b). Data were beamformed onto a $6 \mathrm{~mm}$ grid using normalized lead fields. Then, source estimates were normalized by the power of the projected sensor noise. Source space data were filtered in alpha (8-13 Hz) and beta bands (13-30 Hz). After obtaining the regional time series on the basis of the Automated Anatomical labeling atlas (AAL)(Tzourio-Mazoyer et al., 2002), a symmetric orthogonalization procedure (Colclough et al., 2015)was performed for signal leakage removal. To ultimately estimate the functional connectivity between regional time series, we used the amplitude envelope correlation measure (AEC) (Brookes et al., 2012). This method briefly consists of 1) computing the power envelopes as the magnitude of the signal, using the Hilbert transform, and 2) measuring the linear amplitude correlation between the logarithms of ROI power envelopes. Finally, a sliding window (length $=6 \mathrm{~s}$, step $=0.5 \mathrm{~s}$ ) was applied to construct the dynamic connectivity matrices. This sliding window has been previously used to reconstruct the dynamic networks derived from MEG data (O'Neill et al., 2017a). Also, matrices were thresholded by keeping the strongest $15 \%$ connections of each network.

\subsection{Extracting modular brain states}

Modularity refers to the extent to which a network can be separated into modules or communities highly intra-connected and weakly interconnected (Sporns and Betzel, 2016). To track the transient changes of the brain modular networks over time, we used our recent proposed algorithm (A. Kabbara et al., 2019) that aims to extract the main modular structures (i.e. modular states) that fluctuate repetitively across time. Each modular state reflects unique spatial modular organization. Briefly, the algorithm consists of applying the following steps:

- Decompose each temporal network into modules. As different modularity algorithm may lead to different modules (due to the degeneracy problem), we aimed to Combine the results of the commonly used modularity algorithms: Girvan-Newman (Girvan and Newman, 2002) and Louvain algorithm (Blondel et al., 2008). Each modularity algorithm was also repeated for 200 iterations, as we are aware that the degeneracy problem is present across different runs. This process will result, for each network, in 400 modular organizations (200 runs x 2 algorithms). To define the final modular organization, we performed the consensus algorithm proposed by (Bassett et al., 2013) which consists of computing an association matrix of $\mathrm{N} \mathrm{x} \mathrm{N}$ (where $\mathrm{N}$ is the number of nodes) by counting the number of times two nodes are assigned to the same module across the 400 modular organizations obtained across the 200 runs and the two modularity algorithms. The association matrix is then compared to a null model association matrix computed from random permutations of the original partitions. After this comparison, only the significant values of the association matrix were retained. The thresholded association matrix was finally clustered using Louvain algorithm repeated for 100 iterations. The consensus approach applied on the association matrix was robust across multiple runs leading to a very low quality of consensus.

- Assess the similarity between the temporal modular structures using the z-score of Rand coefficient, a value between 0 (totally different structures) and 1 (identical structures) as proposed by (Traud et al., 2008). This step generated a T $x \mathrm{~T}$ similarity matrix where $\mathrm{T}$ is the number of time windows.

- Cluster the similarity matrix into modular states (MS) using the consensus modularity method. This step associates common temporal modular structures into the same state. Hence, a single community structure was derived from each MS. This was done by forming an association 
matrix from the temporal modular affiliations corresponding to the MS followed by applying the consensus algorithm on the obtained association matrix.

\subsection{Extract the modules associated with RSNs}

Our objective is to characterize the dynamic behavior of spontaneous brain networks in terms of merging and splitting across and within resting state networks. For this reason, we evaluated the degree of resemblance between each module (derived from all the MSs) and the RSNs. To do that, we formed different masks or templates; where each mask is related to a RSN, a subpart of a RSN or a combination between different RSNs. Then, an overall match for each module with each template is calculated. If the overlap between the module and a template is higher than $80 \%$, the module is ultimately associated to the considered template. This overlap is calculated using the following formula:

Overlap $_{m, R S N}=\frac{N \text { common }}{2 * N 1}+\frac{N \text { common }}{2 * N 2}$

Where Ncommon is the number of common nodes between the module $\mathrm{m}$ and the RSN, $N 1$ is the total number of nodes in the module $\mathrm{m}, N 2$ is the total number of nodes in the RSN.

The definition of the RSN templates were mainly based on a previous study described by Shirer et al. (Shirer et al., 2012) in which functional networks were identified: (anterior/posterior salience network, auditory network, dorsal/ventral default mode network, higher/primary visual network, language network, left/right executive control network..). We also added other RSNs based on previous functional resting-state studies: left/right temporal networks and the dorsal attentional network (Allen et al., 2017; E.A. 2014; Baker et al., 2014; Damoiseaux et al., 2006; Fox and Raichle, 2007; Greicius et al., 2003). See Table S8 in the supplemental material for the RSNs definition.

This step aims to standardize the extracted communities as "prototype networks (i.e. RSNs)" which allows analyzing the consistency of the derived modules at the group-level and validates the single subject results.

\subsection{Quantification}

For each module revealed to be associated to an RSN template, two metrics were computed:

1 The temporal fractional occupancy (FO) which represents the total time spent by each module as measured by percentage. Thus, a high value of FO reflects high temporal dominance of the module.

2 The mean dwell time (DT) defined as the average number of consecutive windows spent in a specific module. A module with a high DT is thus considered as a stable or "steady" module, compared to modules with low DT that are considered as "transient" modules.

\subsection{Statistical tests}

In order to investigate whether the observed brain modules are related to subjective internal thoughts and feelings experienced during resting-state acquisition, we have assessed the statistical relationships between the occurrence of modules and the phenotypes of cognition measured by the Resting-State Questionnaire (rsQ). More specifically, Pearson's correlation between the five main indices derived from the rsQ (i.e. visual mental imagery, inner language, somatosensory awareness, inner musical experience, and mental manipulation of numbers) and the fractional occupancies of the derived modules were computed for the 57 participants provided by Dataset 2 . To consider the multiple comparisons problem (between the five types of mental activity, and the 11 modules), $p$-values were corrected using Bonferroni procedure (Bland and Altman, 1995) yielding an adjusted threshold of $p<0.0009$.

\section{Code availability}

Data pre-processing was done using automagic Matlab toolbox https://github.com/methlabUZH/automagic (Pedroni et al., 2019) for dataset 1. Brainstorm toolbox (Tadel et al., 2011) was used to pre-process the signals of dataset 2 , and to reconstruct the regional time series using wMNE. To estimate the head model, OpenMEEG (Gramfort et al., 2010) software was used. Brain Networks estimation of EEG data (datasets 1,2) was done using Matlab. Beamforming construction and networks estimation of MEG data (dataset 3) was performed using the megconnectome pipeline package https://www.humanconnectome. org/software/hcp-meg-pipelines. The Matlab code developed to extract the modular brain states is publicly available at https://github.com/ librteam/Modularity_algorithm_NN. BrainNetViewer (BNV) (Xia et al., 2013) https://www.nitrc.org/projects/bnv/ was used for networks visualization. Other homemade codes were also developed for statistical tests, and quantitative evaluation.

\section{Data availability}

The data used here are all available. The dataset 1 can be found on http://fcon_1000.projects.nitrc.org/indi/cmi_healthy_brain_network/ sharing_neuro.html, the dataset 2 can be available upon a simple request to the correspondent author and the dataset 3 is available on https://db.humanconnectome.org/.

\section{Credit author statement}

Aya Kabbara: Conceptualization, Methodology, Software, Writing, Original draft preparation. Mahmoud Hassan: Conceptualization, Methodology, Writing, Supervision, Funding acquisition. Veronique Paban: Data collection (dataset 3), Resources.

\section{Acknowledgments}

This work was financed by the Rennes University, the Institute of Clinical Neuroscience of Rennes (Project named EEGCog). The study was also funded by the National Council for Scientific Research (CNRS) in Lebanon. The authors would also like to thank the Lebanese Association for Scientific Research (LASER) and Campus France, Programme Hubert Curien CEDRE (Project No. 42257YA), for supporting this study.

\section{Supplementary material}

Supplementary material associated with this article can be found, in the online version, at $10.1016 / \mathrm{j}$.neuroimage.2020.117674.

\section{References}

Agcaoglu, O., Wilson, T.W., Wang, Y.P., Stephen, J., Calhoun, V.D., 2019. Resting state connectivity differences in eyes open versus eyes closed conditions. Hum. Brain Mapp. doi:10.1002/hbm. 24539 .

Alexander, L.M., Escalera, J., Ai, L., Andreotti, C., Febre, K., Mangone, A., Vega-Potler, N., Langer, N., Alexander, A., Kovacs, M., Litke, S., O' Hagan, B., Andersen, J., Bronstein, B., Bui, A., Bushey, M., Butler, H., Castagna, V., Camacho, N., Chan, E., Citera, D., Clucas, J., Cohen, S., Dufek, S., Eaves, M., Fradera, B., Gardner, J., GrantVillegas, N., Green, G., Gregory, C., Hart, E., Harris, S., Horton, M., Kahn, D., Kabotyanski, K., Karmel, B., Kelly, S.P., Kleinman, K., Koo, B., Kramer, E., Lennon, E., Lord, C., Mantello, G., Margolis, A., Merikangas, K.R., Milham, J., Minniti, G., Neuhaus, R., Levine, A., Osman, Y., Parra, L.C., Pugh, K.R., Racanello, A., Restrepo, A., Saltzman, T., Septimus, B., Tobe, R., Waltz, R., Williams, A., Yeo, A., Castellanos, F.X., Klein, A., Paus, T., Leventhal, B.L., Craddock, R.C., Koplewicz, H.S., Milham, M.P., 2017. Data descriptor: an open resource for transdiagnostic research in pediatric mental health and learning disorders. Sci. Data. doi:10.1038/sdata.2017.181.

Allen, E.A., Damaraju, E., Eichele, T., Wu, L., Calhoun, V.D., 2017. EEG signatures of dynamic functional network connectivity states. Brain Topogr. 1-16. doi:10.1007/s10548-017-0546-2.

Allen, E.A., Damaraju, E., Plis, S.M., Erhardt, E.B., Eichele, T., Calhoun, V.D., 2014. Tracking whole-brain connectivity dynamics in the resting state. Cereb. Cortex 24, 663-676. doi:10.1093/cercor/bhs352. 
Andrews-Hanna, J.R., Snyder, A.Z., Vincent, J.L., Lustig, C., Head, D., Raichle, M.E., Buckner, R.L., 2007. Disruption of large-scale brain systems in advanced aging. Neuron doi:10.1016/j.neuron.2007.10.038.

Baker, A.P., Brookes, M.J., Rezek, I.A., Smith, S.M., Behrens, T., Smith, P.J.P., Woolrich, M., 2014. Fast transient networks in spontaneous human brain activity. ELIFE 2014. doi:10.7554/eLife.01867.

Bassett, D.S., Porter, M.A., Wymbs, N.F., Grafton, S.T., Carlson, J.M., Mucha, P.J., 2013. Robust detection of dynamic community structure in networks. Chaos 23 . doi:10.1063/1.4790830.

Bassett, D.S., Sporns, O., 2017. Network neuroscience. Nat. Neurosci. 20, 353-364. doi:10.1038/nn.4502.

Bassett, D.S., Wymbs, N.F., Porter, M.A., Mucha, P.J., Carlson, J.M., Grafton, S.T., 2011. Dynamic reconfiguration of human brain networks during learning. Proc. Natl. Acad. Sci. U. S. A. 108, 7641-7646. doi:10.1073/pnas.1018985108.

Bassett, D.S., Yang, M., Wymbs, N.F., Grafton, S.T., 2015. Learning-induced autonomy of sensorimotor systems. Nat. Neurosci. 18, 744-751. doi:10.1038/nn.3993.

Bernas, A., Barendse, E.M., Aldenkamp, A.P., Backes, W.H., Hofman, P.A.M., Hendriks, M.P.H., Kessels, R.P.C., Willems, F.M.J., de With, P.H.N., Zinger, S., Jansen, J.F.A., 2018. Brain resting-state networks in adolescents with highfunctioning autism: analysis of spatial connectivity and temporal neurodynamics. Brain Behav. doi:10.1002/brb3.878.

Bland, j.M., Altman, D.G., 1995. Multiple significance tests: the Bonferroni method. BMJ doi:10.1136/bmj.310.6973.170.

Blondel, V.D., Guillaume, J.-.L., Lambiotte, R., Lefebvre, E., 2008. Fast unfolding of communities in large networks. J. Stat. Mech. Theory Exp. 10008, 6. doi:10.1088/1742-5468/2008/10/P10008.

Brookes, M.J., Woolrich, M., Luckhoo, H., Price, D., Hale, J.R., Stephenson, M.C., Barnes, G.R., Smith, S.M., Morris, P.G., 2011. Investigating the electrophysiological basis of resting state networks using magnetoencephalography. Proc. Natl. Acad. Sci. U. S. A. 108, 16783-16788. doi:10.1073/pnas.1112685108.

Brookes, M.J., Woolrich, M.W., Barnes, G.R., 2012. Measuring functional connectivity in MEG: a multivariate approach insensitive to linear source leakage. Neuroimage 63, 910-920. doi:10.1016/j.neuroimage.2012.03.048.

Colclough, G.L., Brookes, M.J., Smith, S.M., Woolrich, M.W., 2015. A symmetric multivariate leakage correction for MEG connectomes. Neuroimage 117, 439-448. doi:10.1016/j.neuroimage.2015.03.071.

Colclough, G.L., Woolrich, M.W., Tewarie, P.K., Brookes, M.J., Quinn, A.J., Smith, S.M., 2016a. How reliable are MEG resting-state connectivity metrics? Neuroimage 138, 284-293. doi:10.1016/j.neuroimage.2016.05.070.

Colclough, G.L., Woolrich, M.W., Tewarie, P.K., Brookes, M.J., Quinn, A.J., Smith, S.M., 2016b. How reliable are MEG resting-state connectivity metrics? Neuroimage 138, 284-293. doi:10.1016/j.neuroimage.2016.05.070.

Coquelet, N., De Tiège, X., Destoky, F., Roshchupkina, L., Bourguignon, M., Goldman, S., Peigneux, P., Wens, V., 2020. Comparing MEG and highdensity EEG for intrinsic functional connectivity mapping. Neuroimage, 116556 doi:10.1016/J.NEUROIMAGE.2020.116556.

Damoiseaux, J.S., Prater, K.E., Miller, B.L., Greicius, M.D., 2012. Functional connectivity tracks clinical deterioration in Alzheimer's disease. Neurobiol. Aging 33. doi:10.1016/j.neurobiolaging.2011.06.024.

Damoiseaux, J.S., Rombouts, S.A.R.B., Barkhof, F., Scheltens, P., Stam, C.J., Smith, S.M., Beckmann, C.F., 2006. Consistent resting-state networks across healthy subjects. Proc. Natl. Acad. Sci. U. S. A. 103, 13848-13853. doi:10.1073/pnas.0601417103.

de Pasquale, F., Corbetta, M., Betti, V., Della Penna, S., 2018. Cortical cores in network dynamics. Neuroimage doi:10.1016/j.neuroimage.2017.09.063.

de Pasquale, F., Penna, S.Della, Sporns, O., Romani, G.L., Corbetta, M., 2015. A dynamic core network and global efficiency in the resting human brain. Cereb. Cortex bhv185. 10.1093/cercor/bhv185.

Delamillieure, P., Doucet, G., Mazoyer, B., Turbelin, M.R., Delcroix, N., Mellet, E., Zago, L., Crivello, F., Petit, L., Tzourio-Mazoyer, N., Joliot, M., 2010. The resting state questionnaire: an introspective questionnaire for evaluation of inner experience during the conscious resting state. Brain Res. Bull. doi:10.1016/j.brainresbull.2009.11.014.

Desikan, R.S., Sugonne, F., Fischl, B., Quinn, B.T., Dickerson, B.C., Blacker, D., Buckner, R.L., Dale, A.M., Maguire, R.P., Hyman, B.T., Albert, M.S., Killiany, R.J., 2006. An automated labeling system for subdividing the human cerebral cortex on MRI scans into gyral based regions of interest. Neuroimage 31, 968-980. doi:10.1016/j.neuroimage.2006.01.021.

Diaz, B.A., Hardstone, R., Mansvelder, H.D., Van Someren, E.J.W., Linkenkaer-Hansen, K., 2016. Resting-state subjective experience and EEG biomarkers are associated with sleep-onset latency. Front. Psychol. doi:10.3389/fpsyg.2016.00492.

Doucet, G., Naveau, M., Petit, L., Zago, L., Crivello, F., Jobard, G., Delcroix, N., Mellet, E., Tzourio-Mazoyer, N., Mazoyer, B., Joliot, M., 2012. Patterns of hemodynamic lowfrequency oscillations in the brain are modulated by the nature of free thought during rest. Neuroimage doi:10.1016/j.neuroimage.2011.11.059.

Filippi, M., Spinelli, E.G., Cividini, C., Agosta, F., 2019. Resting state dynamic functional connectivity in neurodegenerative conditions: a review of magnetic resonance imaging findings. Front. Neurosci. doi:10.3389/fnins.2019.00657.

Fox, M.D., Raichle, M.E., 2007. Spontaneous fluctuations in brain activity observed with functional magnetic resonance imaging. Nat. Rev. Neurosci. 8, 700-711 nrn2201 [pii] $\backslash n 10.1038 / \mathrm{nrn} 2201$.

Garrison, K.A., Scheinost, D., Finn, E.S., Shen, X., Constable, R.T., 2015. The (in)stability of functional brain network measures across thresholds. Neuroimage 118, 651-661. doi:10.1016/j.neuroimage.2015.05.046.

Girvan, M., Newman, M.E.J., 2002. Community structure in social and biological networks. Proc. Natl. Acad. Sci. U. S. A. 99, 7821-7826. doi:10.1073/pnas.122653799.
Gramfort, A., Papadopoulo, T., Olivi, E., Clerc, M., 2010. OpenMEEG: opensource software for quasistatic bioelectromagnetics. Biomed. Eng. Online 9. doi:10.1186/1475-925X-8-1.

Greicius, M.D., Krasnow, B., Reiss, A.L., Menon, V., 2003. Functional connectivity in the resting brain: a network analysis of the default mode hypothesis. Proc. Natl. Acad. Sci. U. S. A. 100, 253-258. doi:10.1073/pnas.0135058100.

Hamalainen, M.S., Ilmoniemi, R.J., 1994. Interpreting magnetic fields of the brain: minimum norm estimates. Med. Biol. Eng. Comput. 32, 35-42. doi:10.1007/BF02512476.

Hassan, M., Dufor, O., Merlet, I., Berrou, C., Wendling, F., 2014. EEG source connectivity analysis: from dense array recordings to brain networks. PLoS ONE 9. doi:10.1371/journal.pone.0105041.

Hassan, M., Merlet, I., Mheich, A., Kabbara, A., Biraben, A., Nica, A., Wendling, F., 2016. Identification of interictal epileptic networks from dense-EEG. Brain Topogr 1-17. doi:10.1007/s10548-016-0517-z.

Hassan, Mahmoud, Wendling, F., 2018. Electroencephalography source connectivity: aiming for high resolution of brain networks in time and space. IEEE Signal Process. Mag. 35, 81-96. doi:10.1109/MSP.2017.2777518.

Hipp, J.F., Hawellek, D.J., Corbetta, M., Siegel, M., Engel, A.K., 2012. Large-scale cortical correlation structure of spontaneous oscillatory activity. Nat. Neurosci. 15, 884-890. doi:10.1038/nn.3101.

Honey, C.J., Kötter, R., Breakspear, M., Sporns, O., 2007. Network structure of cerebral cortex shapes functional connectivity on multiple time scales. Proc. Natl. Acad. Sci. U. S. A. doi:10.1073/pnas.0701519104.

Hyvärinen, A., Hirayama, J.I., Kiviniemi, V., Kawanabe, M., 2016. Orthogonal connectivity factorization: interpretable decomposition of variability in correlation matrices. Neural Comput. doi:10.1162/NECO_a_00810.

Jiao, Z., Wang, H., Cai, M., Cao, Y., Zou, L., Wang, S., 2018. Rich club characteristics of dynamic brain functional networks in resting state. Multimed. Tools Appl. doi:10.1007/s11042-018-6424-4.

Jones, D.T., Vemuri, P., Murphy, M.C., Gunter, J.L., Senjem, M.L., Machulda, M.M., Przybelski, S.A., Gregg, B.E., Kantarci, K., Knopman, D.S., Boeve, B.F., Petersen, R.C. Jack, C.R., 2012. Non-stationarity in the "resting brain's" modular architecture. PLoS ONE doi:10.1371/journal.pone.0039731.

Kabbara, A., Eid, H., El Falou, W., Khalil, M., Wendling, F., Hassan, M., 2018. Reduced integration and improved segregation of functional brain networks in Alzheimer's disease. J. Neural Eng. 15. doi:10.1088/1741-2552/aaaa76.

Kabbara, A., Falou, W.E.L., Khalil, M., Wendling, F., Hassan, M., 2017. The dynamic functional core network of the human brain at rest. Sci. Rep. 7, 2936.

Kabbara, A., Khalil, M., O’Neill, G., Dujardin, K., El Traboulsi, Y., Wendling, F., Hassan, M., 2019. Detecting modular brain states in rest and task. Netw. Neurosci. doi:10.1162/netn_a_00090.

Kabbara, A., Paban, V., Weill, A., Modolo, J., Hassan, M., 2020. Brain network dynamics correlate with personality traits. Brain Connect doi:10.1089/brain.2019.0723.

Kenett, Y.N., Betzel, R.F., Beaty, R.E., 2020. Community structure of the creative brain at rest. Neuroimage 210, 116578. doi:10.1016/J.NEUROIMAGE.2020.116578.

Kitzbichler, M.G., Henson, R.N.A., Smith, M.L., Nathan, P.J., Bullmore, E.T., 2011. Cognitive effort drives workspace configuration of human brain functional networks. J. Neurosci. doi:10.1523/JNEUROSCI.0440-11.2011.

Lachaux, J.-.P., Rodriguez, E., Le van Quyen, M., Lutz, A., Martinerie, J., Varela, F.J., 2000. Studying single-trials of phase synchronous activity in the brain. Int. J. Bifurc. Chaos 10, 2429-2439. doi:10.1142/S0218127400001560.

Leonardi, N., Richiardi, J., Gschwind, M., Simioni, S., Annoni, J.M., Schluep, M., Vuilleumier, P., Van De Ville, D., 2013. Principal components of functional connectivity: a new approach to study dynamic brain connectivity during rest. Neuroimage doi:10.1016/j.neuroimage.2013.07.019.

Liu, C., Xue, J., Cheng, X., Zhan, W., Xiong, X., Wang, B., 2019. Tracking the brain state transition process of dynamic function connectivity based on resting state fmri. Comput. Intell. Neurosci. doi:10.1155/2019/9027803.

Liu, Z., Fukunaga, M., de Zwart, J.A., Duyn, J.H., 2010. Large-scale spontaneous fluctuations and correlations in brain electrical activity observed with magnetoencephalography. Neuroimage 51, 102-111. doi:10.1016/j.neuroimage.2010.01.092.

Meunier, D., Achard, S., Morcom, A., Bullmore, E., 2009. Age-related changes in modular organization of human brain functional networks. Neuroimage 44, 715-723. doi:10.1016/j.neuroimage.2008.09.062.

Moussa, M.N., Vechlekar, C.D., Burdette, J.H., Steen, M.R., Hugenschmidt, C.E., Laurienti, P.J., 2011. Changes in cognitive state alter human functional brain networks. Front. Hum. Neurosci. 5, 1-15. doi:10.3389/fnhum.2011.00083.

O'Neill, G.C., Tewarie, P., Vidaurre, D., Liuzzi, L., Woolrich, M.W., Brookes, M.J., 2017a. Dynamics of large-scale electrophysiological networks: a technical review. Neuroimage doi:10.1016/j.neuroimage.2017.10.003.

O'Neill, G.C., Tewarie, P.K., Colclough, G.L., Gascoyne, L.E., Hunt, B.A.E., Morris, P.G., Woolrich, M.W., Brookes, M.J., 2017b. Measurement of dynamic task related functional networks using MEG. Neuroimage 146, 667-678. doi:10.1016/j.neuroimage.2016.08.061.

Owen, J.P., Li, Y.O., Yang, F.G., Shetty, C., Bukshpun, P., Vora, S., Wakahiro, M., Hinkley, L.B.N., Nagarajan, S.S., Sherr, E.H., Mukherjee, P., 2013. Resting-state networks and the functional connectome of the human brain in agenesis of the corpus callosum. Brain Connect doi:10.1089/brain.2013.0175.

Paban, V., Modolo, J., Mheich, A., Hassan, M., 2019. Psychological resilience correlates with EEG source-space brain network flexibility. Netw. Neurosci. doi:10.1162/netn_a_00079.

Patriat, R., Molloy, E.K., Meier, T.B., Kirk, G.R., Nair, V.A., Meyerand, M.E., Prabhakaran, V., Birn, R.M., 2013. The effect of resting condition on resting-state fMR 
reliability and consistency: a comparison between resting with eyes open, closed, and fixated. Neuroimage doi:10.1016/j.neuroimage.2013.04.013.

Pedroni, A., Bahreini, A., Langer, N., 2019. Automagic: standardized preprocessing of big EEG data. Neuroimage doi:10.1016/j.neuroimage.2019.06.046.

Pipinis, E., Melynyte, S., Koenig, T., Jarutyte, L., Linkenkaer-Hansen, K., Ruksenas, O., Griskova-Bulanova, I., 2017. Association between resting-state microstates and ratings on the amsterdam resting-state questionnaire. Brain Topogr. doi:10.1007/s10548-016-0522-2.

Preti, M.G., Van De Ville, D., 2016. Eigenmaps of dynamic functional connectivity: voxellevel dominant patterns through eigenvector centrality. In: Proceedings - International Symposium on Biomedical Imaging doi:10.1109/ISBI.2016.7493431.

Raichle, M.E., MacLeod, A.M., Snyder, A.Z., Powers, W.J., Gusnard, D.A., Shulman, G.L., 2001. A default mode of brain function. Proc. Natl. Acad. Sci. U. S. A. 98, 676-682. doi:10.1073/pnas.98.2.676.

Rasero, J., Pellicoro, M., Angelini, L., Cortes, J.M., Marinazzo, D., Stramaglia, S., 2017. Consensus clustering approach to group brain connectivity matrices. Netw. Neurosci. 1, 242-253. doi:10.1162/NETN_a 00017.

Schoffelen, J.M., Gross, J., 2009. Source connectivity analysis with MEG and EEG. Hum. Brain Mapp. doi:10.1002/hbm.20745.

Shirer, W.R., Ryali, S., Rykhlevskaia, E., Menon, V., Greicius, M.D., 2012. Decoding subject-driven cognitive states with whole-brain connectivity patterns. Cereb. Cortex 22, 158-165. doi:10.1093/cercor/bhr099.

Sporns, O., Betzel, R.F., 2016. Modular brain networks. Annu. Rev. Psychol. 67, 613-640. doi:10.1146/annurev-psych-122414-033634.

Stoffers, D., Diaz, B.A., Chen, G., Den Braber, A., Van't Ent, D., Boomsma, D.I., Mansvelder, H.D., De Geus, E., Van Someren, E.J.W., Linkenkaer-Hansen, K., 2015. Resting-state fMRI functional connectivity is associated with sleepiness, imagery, and discontinuity of mind. PLoS ONE doi:10.1371/journal.pone.0142014.

Tadel, F., Baillet, S., Mosher, J.C., Pantazis, D., Leahy, R.M., 2011. Brainstorm: a user-friendly application for MEG/EEG analysis. Comput. Intell. Neurosci. 2011. doi:10.1155/2011/879716.

Tijms, B.M., Wink, A.M., de Haan, W., van der Flier, W.M., Stam, C.J., Scheltens, P., Barkhof, F., 2013. Alzheimer's disease: connecting findings from graph theoretical studies of brain networks. Neurobiol. Aging. doi:10.1016/j.neurobiolaging.2013.02.020.

Tompson, S.H., Falk, E.B., Vettel, J.M., Bassett, D.S., 2018. Network approaches to understand individual differences in brain connectivity: opportunities for personality neuroscience. Personal. Neurosci. doi:10.1017/pen.2018.4.

Traud, A.L., Kelsic, E.D., Mucha, P.J., Porter, M.A., 2008. Comparing community structure to characteristics in online collegiate social networks 53, 526-543. doi: $10.1137 / 080734315$.
Tzourio-Mazoyer, N., Landeau, B., Papathanassiou, D., Crivello, F., Etard, O., Delcroix, N., Mazoyer, B., Joliot, M., 2002. Automated anatomical labeling of activations in SPM using a macroscopic anatomical parcellation of the MNI MRI single-subject brain. Neuroimage doi:10.1006/nimg.2001.0978.

Van De Ville, D., Britz, J., Michel, C.M., 2010. EEG microstate sequences in healthy humans at rest reveal scale-free dynamics. Proc. Natl. Acad. Sci. U. S. A. doi:10.1073/pnas.1007841107.

van den Heuvel, M.P., Sporns, O., 2013. Network hubs in the human brain. Trends Cogn. Sci. doi:10.1016/j.tics.2013.09.012.

Van Dijk, K.R.A., Hedden, T., Venkataraman, A., Evans, K.C., Lazar, S.W., Buckner, R.L., 2010. Intrinsic functional connectivity as a tool for human connectomics: theory, properties, and optimization. J. Neurophysiol. doi:10.1152/jn.00783.2009.

Van Essen, D.C., Ugurbil, K., Auerbach, E., Barch, D., Behrens, T.E.J., Bucholz, R., Chang, A., Chen, L., Corbetta, M., Curtiss, S.W., Della Penna, S., Feinberg, D. Glasser, M.F., Harel, N., Heath, A.C., Larson-Prior, L., Marcus, D., Michalareas, G., Moeller, S., Oostenveld, R., Petersen, S.E., Prior, F., Schlaggar, B.L., Smith, S.M., Snyder, A.Z., Xu, J., Yacoub, E., 2012. The human connectome project: a data acquisition perspective. Neuroimage doi:10.1016/j.neuroimage.2012.02.018.

Van Veen, B.D., Van Drongelen, W., Yuchtman, M., Suzuki, A., 1997. Localization of brain electrical activity via linearly constrained minimum variance spatial filtering. IEEE Trans. Biomed. Eng. doi:10.1109/10.623056.

Vidaurre, D., Hunt, L.T., Quinn, A.J., Hunt, B.A.E., Brookes, M.J., Nobre, A.C., Woolrich, M.W., 2018. Spontaneous cortical activity transiently organises into frequency specific phase-coupling networks. Nat. Commun. doi:10.1038/s41467-018-05316-z.

Wens, V., Bourguignon, M., Vander Ghinst, M., Mary, A., Marty, B., Coquelet, N., Naeije, G., Peigneux, P., Goldman, S., De Tiège, X., 2019. Synchrony, metastability, dynamic integration, and competition in the spontaneous functional connectivity of the human brain. Neuroimage 199, 313-324. doi:10.1016/J.NEUROIMAGE.2019.05.081.

Winkler, I., Haufe, S., Tangermann, M., 2011. Automatic classification of artifactual ICA-components for artifact removal in EEG signals. Behav. Brain Funct. doi:10.1186/1744-9081-7-30.

Xia, M., Wang, J., He, Y., 2013. BrainNet viewer: a network visualization tool for human brain connectomics. PLoS ONE 8. doi:10.1371/journal.pone.0068910.

Yan, C., Liu, D., He, Y., Zou, Q., Zhu, C., Zuo, X., Long, X., Zang, Y., 2009. Spontaneous brain activity in the default mode network is sensitive to different resting-state conditions with limited cognitive load. PLoS ONE doi:10.1371/journal.pone.0005743.

Zalesky, A., Fornito, A., Cocchi, L., Gollo, L.L., Breakspear, M., 2014. Timeresolved resting-state brain networks. Proc. Natl. Acad. Sci. U. S. A. doi:10.1073/pnas.1400181111. 